\title{
乌s \\ Phase errors and their effect on undulator radiation properties
}

\author{
Richard P. Walker \\ Diamond Light Source, Oxfordshire, United Kingdom \\ (Received 3 November 2012; published 22 January 2013; corrected 20 February 2013)
}

\begin{abstract}
A detailed analysis is carried out of the various types of phase errors present in real undulator devices, and their statistical properties. The influence of phase errors on the radiation properties is also examined, distinguishing the effects on peak brightness and integrated flux, and including the effects of electron beam emittance and energy spread. The limitation of the usual expression for the reduction in intensity due to phase errors, based on the rms phase error, is explored, and a new parameter is introduced which correlates better with the reduction in integrated flux. The implications for operation of undulators in future lower emittance storage rings is also discussed.
\end{abstract}

DOI: 10.1103/PhysRevSTAB.16.010704

PACS numbers: 41.60.Ap

\section{INTRODUCTION}

Undulators are the predominant sources of radiation in modern synchrotron light sources. The influence of magnetic field errors on their performance, as well as methods for correcting them in order to optimize their performance, have been the subject of investigation for over 25 years. In 1985 , pioneering work by Kincaid showed that the main effect of field errors is on the phase term in the radiation emission formula, introducing "phase noise" which can seriously reduce the radiation brightness (angular and spectral flux density) [1]. He derived formulas for the expected reduction in intensity as a function of rms field amplitude error. Diviacco and Walker later showed however that, for a given rms field error, there is a large statistical variation in intensity, which makes this parameter unsuitable as a "figure of merit" to describe undulator quality [2]. A similar conclusion was drawn by Kennedy et al. for low-gain free-electron laser (FEL) performance [3]. Instead, Bobbs et al. showed that both spontaneous emission intensity and FEL small signal gain correlated well with rms "phase shake"[4]. Subsequently, it was shown [5] that the reduction in on-axis brightness due to phase errors could be modeled well by the simple expression,

$$
R=\exp \left(-n^{2} \sigma_{\varphi}^{2}\right),
$$

where $n$ is the harmonic number and $\sigma_{\varphi}$ is the rms phase error at the magnet poles. Once the importance of the radiation phase became appreciated, various techniques were developed for correcting it as part of the undulator field optimization and "shimming" process by many groups, e.g., [6-11]. As a result of these developments it became relatively routine to achieve phase errors of a few

Published by the American Physical Society under the terms of the Creative Commons Attribution 3.0 License. Further distribution of this work must maintain attribution to the author(s) and the published article's title, journal citation, and DOI. degrees, so allowing operation on higher harmonic numbers than originally thought possible. For example, for $\sigma_{\varphi}=2.5^{\circ}$ the reduction in intensity on the 11 th harmonic according to Eq. (1) is only $20 \%$, whereas for the early 3rd generation light sources, only operation up to the 5th harmonic was initially considered feasible.

Although it has been known for some time that the effect of phase errors is less severe than predicted by Eq. (1) when electron beam emittance and energy spread, as well as a realistic range of collection angles, are taken into account, little has been written on the subject (e.g., Ref. [12]). Dejus made a detailed study in 2003, comparing two representative Advanced Photon Source (APS) undulators with "low" $\left(\sigma_{\varphi}=3.7^{\circ}\right)$ and "high" $\left(\sigma_{\varphi}=6.7^{\circ}\right)$ phase errors and concluded that the influence of phase errors is significantly reduced when these effects are included [13]. For example, he showed that, although the ratio of real-to-ideal brightness for the high phase error device is only about $10 \%$ on the 15 th harmonic for the single-electron on-axis case, the ratio improves to $65 \%$ when emittance, energy spread, and pinhole aperture are included. He also showed that the difference between the two devices, although significant for the single-electron on-axis case, becomes marginal when all of these effects are included. More recently, Casalbuoni et al. investigated the effects of emittance, energy spread, and collection angle on the output of a superconducting undulator with a simulated field profile and arrived at a similar conclusion about the weakening of the influence of phase errors [14]. The conclusion of this study was the surprising statement that "a rms phase error of $\sim 6^{\circ}$ is good enough for existing and planned storage rings up to the 15th harmonic".

What these two studies are pointing out is that it may not always be worth the time and effort to reduce the phase error below a certain value (as pointed out in [13]), and this is particularly relevant in the case of a superconducting undulator for which shimming might be impossible, or extremely difficult, which was the context of the observations made in Ref. [14]. The aim of this paper is to 
investigate the reason for the observed reduction of the influence of phase errors in practical cases when electron beam emittance and energy spread, as well as finite collection angles, are included. In Sec. II we first examine the different types of phase error and their statistical properties. In Sec. III we investigate the effects of phase errors on the radiation spectrum, starting with the single-electron, on-axis brightness, and then extending this to include emittance, energy spread, and angular acceptance. Finally, in Sec. IV we consider the implications for future lower emittance storage rings.

\section{CHARACTERISTICS OF PHASE ERRORS}

\section{A. Types of phase errors}

The phase lag between an electron and the front of the emitted wave train, directed along the nominal undulator axis $(z)$, is given by the following:

$$
\phi(z)=\frac{2 \pi}{\lambda}(c t-z)=\frac{2 \pi}{\lambda}\left(\frac{l(z)}{\beta}-z\right)
$$

where $\lambda$ is the radiation wavelength, $\beta$ is the electron's relativistic factor, and $l(z)$ is the electron's path length up to the point $z$. The fundamental wavelength of the undulator radiation is such that over one magnet period the phase lag is $2 \pi$, and hence the emission adds constructively. Phase errors describe the difference between the phase in a real device with field errors, compared to an ideal device, usually with a sinusoidal field distribution. Since the phase is only important at the point of radiation emission at each pole, the phase error is usually evaluated at these points, $\varphi_{i}$, or to be more precise, when the tangent to the trajectory points towards the observer [5].

Phase errors may be divided into three main types. Systematic phase errors vary smoothly along the length of the device, and are due to smoothly varying field amplitude errors, arising from either a variation in magnetic gap, or (less likely) some other parameter such as periodicity or permanent magnet strength. Random phase errors are produced by local magnetic field errors, which vary from pole to pole. Such field errors, in addition to affecting the phase, can also introduce errors in the electron trajectory and the effect of an angular error is to introduce a phase oscillation between positive and negative poles.

Since according to Eq. (2) the phase errors are the result of cumulative path length errors along the device, we can write the phase at any point as the sum of all upstream phase error contributions:

$$
\varphi_{n}=\varphi_{1}+\sum_{i=1}^{n-1} \psi_{i}
$$

where

$$
\psi_{i}=\varphi_{i+1}-\varphi_{i}
$$

In the following the phase error differences, $\psi_{i}$, will also be referred to as the "local phase errors" and $\varphi_{i}$ as simply phase errors, the term "cumulative" being understood. In the case that the $\psi_{i}$ are uncorrelated random errors, then Eq. (3) represents a random-walk process. The simulations of Ref. [5] with random compensated field errors were therefore simulating a random-walk process (although the full significance of this fact was not appreciated at the time).

As a way of analyzing how phase errors are distributed, we introduce the autocorrelation plot, or correlogram, where the autocorrelation coefficients are defined by the following:

$$
c_{h}=\sum_{i=1}^{n_{p}-h} \varphi_{i} \varphi_{i+h} / \sum_{i=1}^{n_{p}} \varphi_{i}^{2},
$$

where $n_{p}$ is the number of poles. Figure 1 shows a purely random set of phase errors $\left(\varphi_{i}\right)$ and its autocorrelation (for illustration, as such a distribution is unlikely to exist in
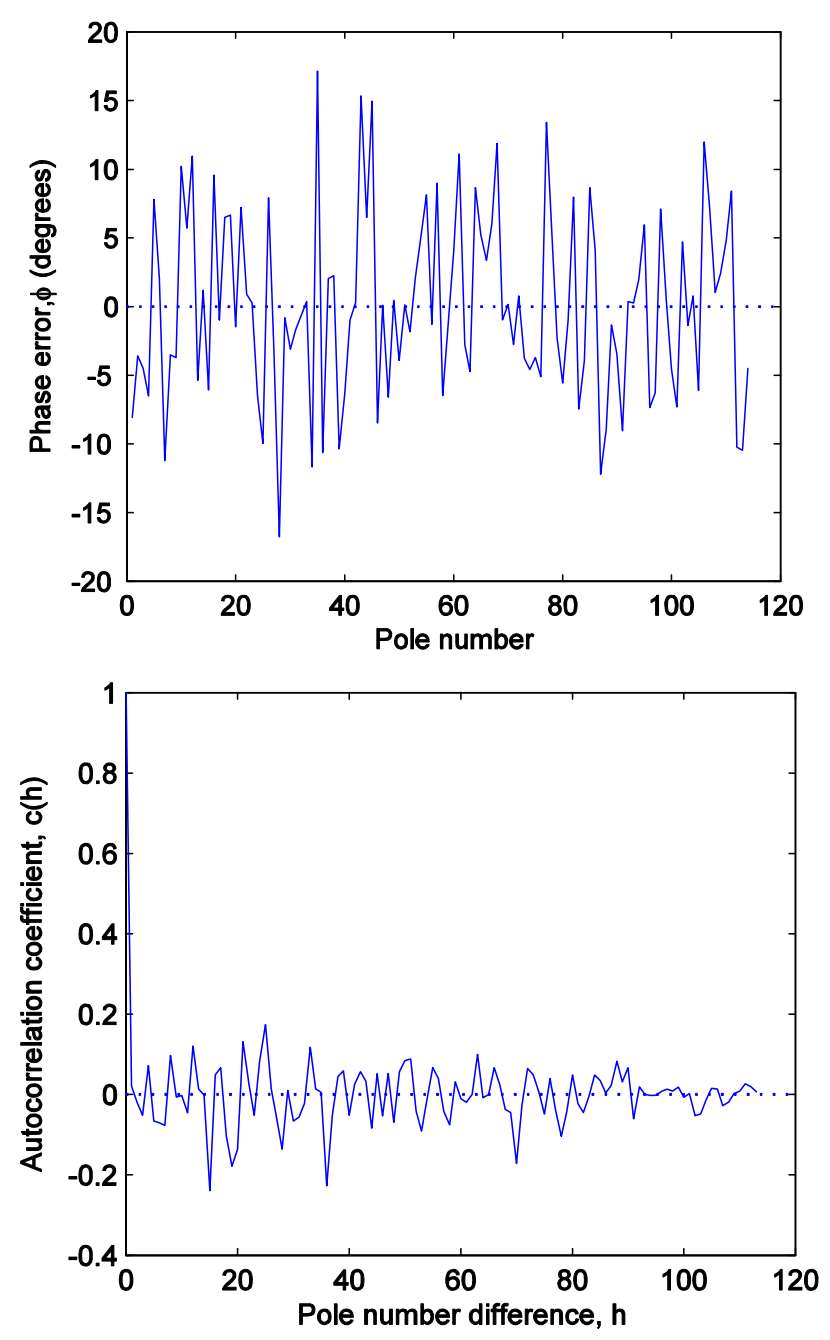

FIG. 1. Phase errors (upper) and corresponding autocorrelation coefficients (lower) for a set of random phase errors with rms value of $\sigma_{\varphi}=6.7^{\circ}$. 
practice) and Fig. 2 the same for a random set of local phase errors $\left(\psi_{i}\right)$, i.e., a random walk. Note that for all sets of phase errors in this report the best-fit straight line has been subtracted as this is simply equivalent to a change in fundamental wavelength. The two cases are clearly very different, the random-walk producing in addition to a random-like local variation, a smooth deviation or "wander" of the phase resulting in significant long-scale correlation, which is absent in the case of independent random errors.

In the case of systematic errors due to smooth variations in magnetic gap, the phase error introduced between two adjacent poles can be approximated by the following:

$$
\psi_{i}=-\pi \frac{K^{2}}{1+K^{2} / 2} \frac{\pi \Delta g_{i}}{\lambda_{o}}
$$
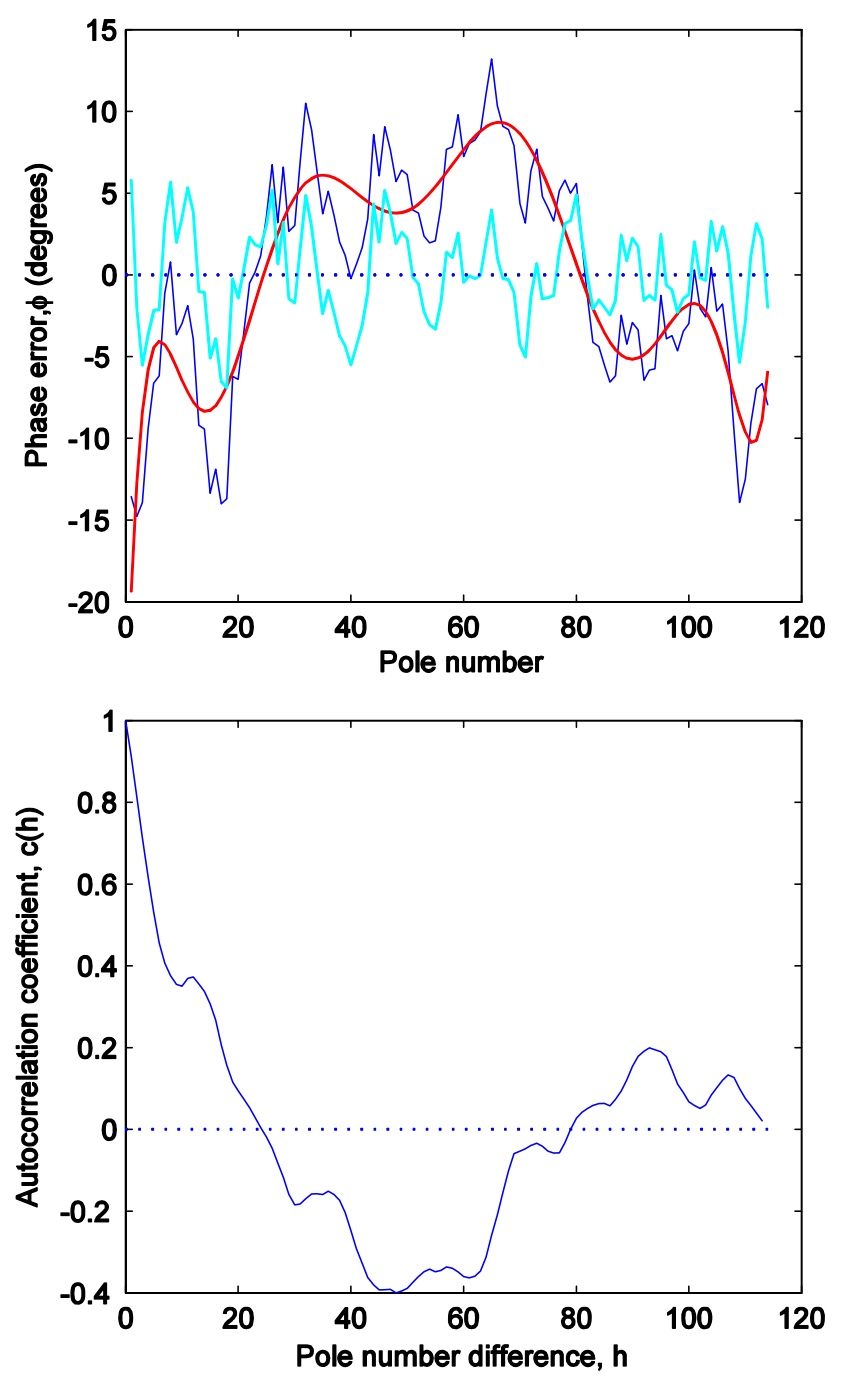

FIG. 2. Phase errors (upper) and corresponding autocorrelation coefficients (lower) for a set of random local phase errors, i.e., a random walk, scaled to give $\sigma_{\varphi}=6.7^{\circ}$. The red and cyan curves show the decomposition into the sum of smooth (red) and random (cyan) distributions. where $\lambda_{o}$ is the period length, $K$ is the usual deflection parameter, and $\Delta g_{i}$ is the gap error in the region of the $i$ th pole. Use has been made of the following approximate relation between field amplitude $B$ and gap: $B=$ $a \exp \left(-\pi g / \lambda_{o}\right)$ from which it follows that $\Delta B / B=$ $-\pi \Delta g / \lambda_{o}$. Figure 3 (upper) shows a number of different gap error functions and the corresponding phase errors (middle plot), scaled to the same rms value as Fig. 2. As would be expected, the autocorrelation function for these cases (lower plot) shows significant correlation is present, not dissimilar in form to those of the random-walk case.

The question of whether these two types of error can be distinguished, when they both produce a smooth variation of phase, is an interesting one. In principle, systematic gap errors produce a smooth variation also in phase difference, whereas they are by definition random for the random-walk errors. The difficulty however is that the phase differences in the case of a smooth gap variation are small compared to the random errors that are usually present. Figure 4 shows the phase differences corresponding to Fig. 2 and one of the cases of Fig. 3, both with the same rms phase error $\sigma_{\varphi}$, and it is clear that the random errors dominate. Thus, whereas in principle the phase differences (or their autocorrelation) might be used to distinguish systematic errors from random errors, this distinction is quickly lost when random errors are present at any significant level. In the next section we will examine this issue again on a statistical basis.

We now look at the influence of trajectory deviations. An angular error introduces a local phase oscillation between positive and negative poles with an amplitude given by $[5,15]$

$$
\delta=\frac{\gamma \theta_{x}}{K} \frac{1+2 K^{2}}{1+K^{2} / 2},
$$

where $\theta_{x}$ is the angle of the trajectory in the horizontal direction (in the usual case with a vertical main field component). The phase is thus very sensitive to angular errors; for example, with a typical $K$ of 2 , an angle of only $2 \mu \mathrm{rad}$ is needed to produce a phase oscillation of $\pm 1^{\circ}$ at $3 \mathrm{GeV}$, at the fundamental. Figure 5 shows phase errors with an rms value of $2^{\circ}$ resulting from a sinusoidal variation of angle along the device. Phase oscillations are clearly distinguishable from both random and smoothly varying phase errors (as well of course as being able to be deduced directly from the field and trajectory data).

\section{B. Phase errors in real devices}

We start by examining the phase errors in the well characterized APS low and high phase error devices [13]. Figure 6 shows the phase errors evaluated at the poles and the corresponding autocorrelation coefficients. The phase errors clearly show phase wander, and the autocorrelation shows clear similarity to that of Fig. 2. Figure 7 shows the phase differences and the respective autocorrelation plots. 

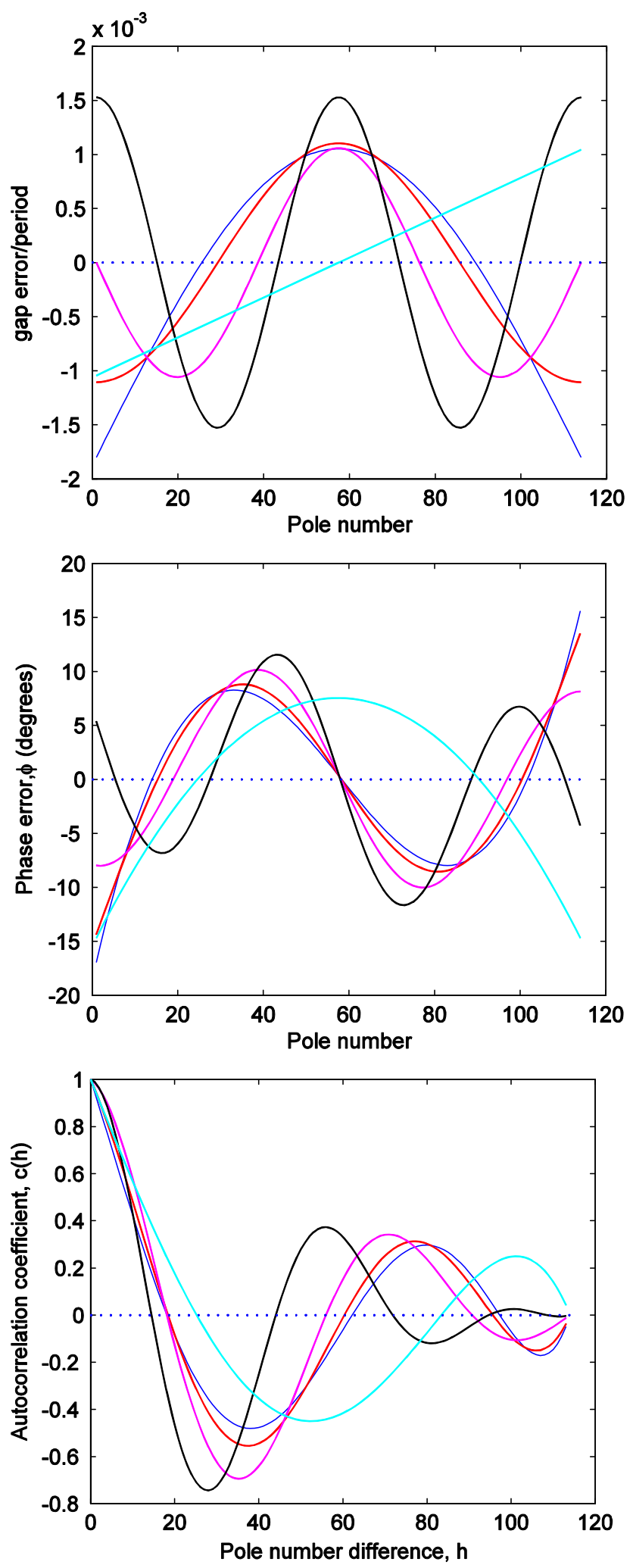

FIG. 3. Gap errors (upper), corresponding phase errors (middle), and their autocorrelation functions (lower) for a linear taper (cyan) and 0.5 (blue), 1 (red), 1.5 (magenta), and 2 (black) periods of a cosine function along the length of a device The gap errors are scaled to produce the same rms phase error in each case, $\sigma_{\varphi}=6.7^{\circ}$.

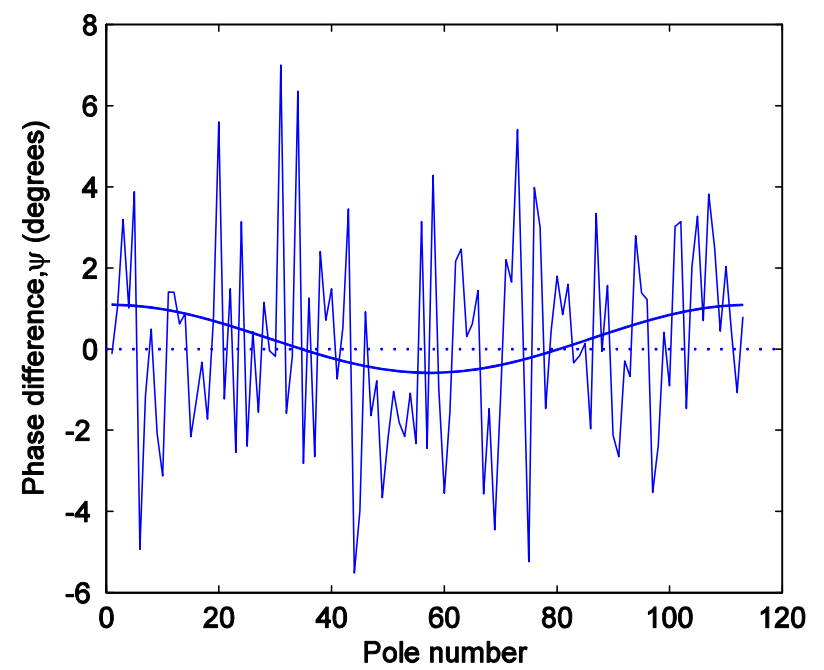

FIG. 4. Phase differences corresponding to Figs. 2 and 3 (red curves).
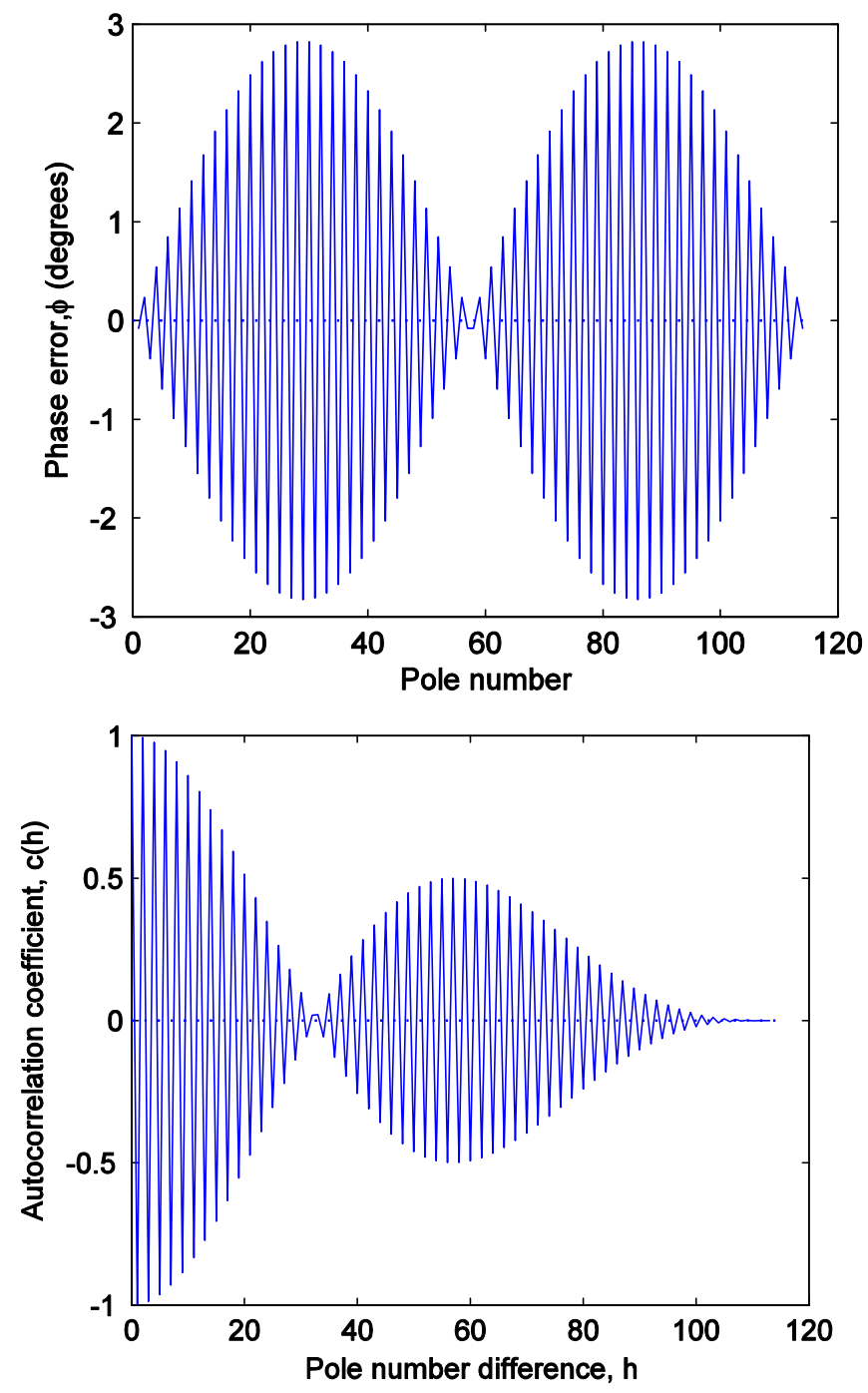

FIG. 5. Phase errors resulting from a sinusoidal trajectory angle error (upper) and the corresponding autocorrelation (lower). 

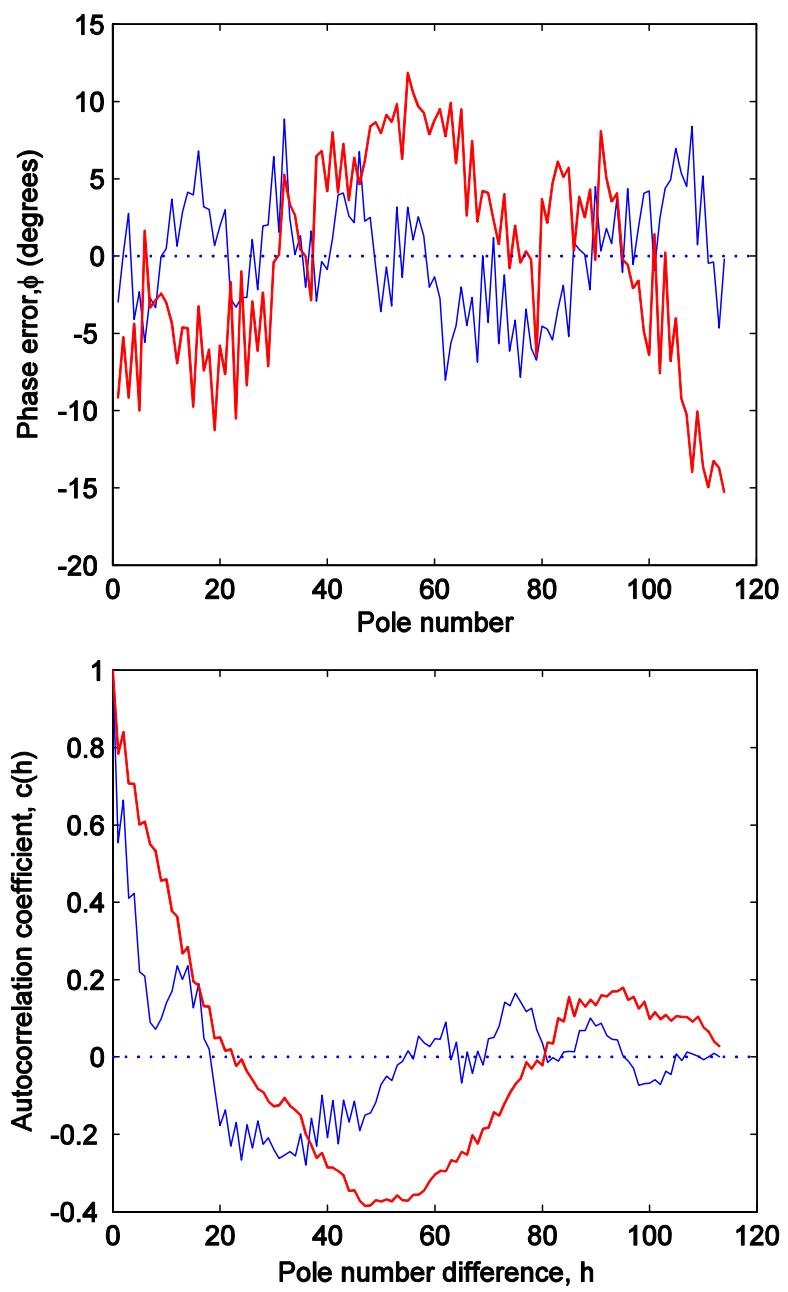

FIG. 6. Phase errors (upper) and autocorrelation coefficients (lower) for the APS low (blue) and high (red) phase error devices.

Now as expected the smooth part of the phase variation is removed, and the autocorrelation reveals some small degree of phase oscillation. Figure 8 shows the differences in phase between next-nearest poles, and its autocorrelation, which removes the phase oscillation and leaves only the random part, and as expected these local phase errors are uncorrelated. In other words, the errors appear to be consistent with a random-walk model, with some small additional phase oscillation. A number of other cases have been examined, all giving similar results: two in-vacuum undulators (before and after shimming) and two APPLE-II devices constructed by Diamond Light Source (DLS), and two of the initial Linac Coherent Light Source (LCLS) undulators [16].

We will now try and examine in more detail the various contributing factors to the phase error. We can estimate the underlying local phase errors by calculating

$$
\sigma_{\psi} \simeq \sqrt{\left\langle\varphi_{i+2}-\varphi_{i}\right\rangle^{2} / 2}
$$
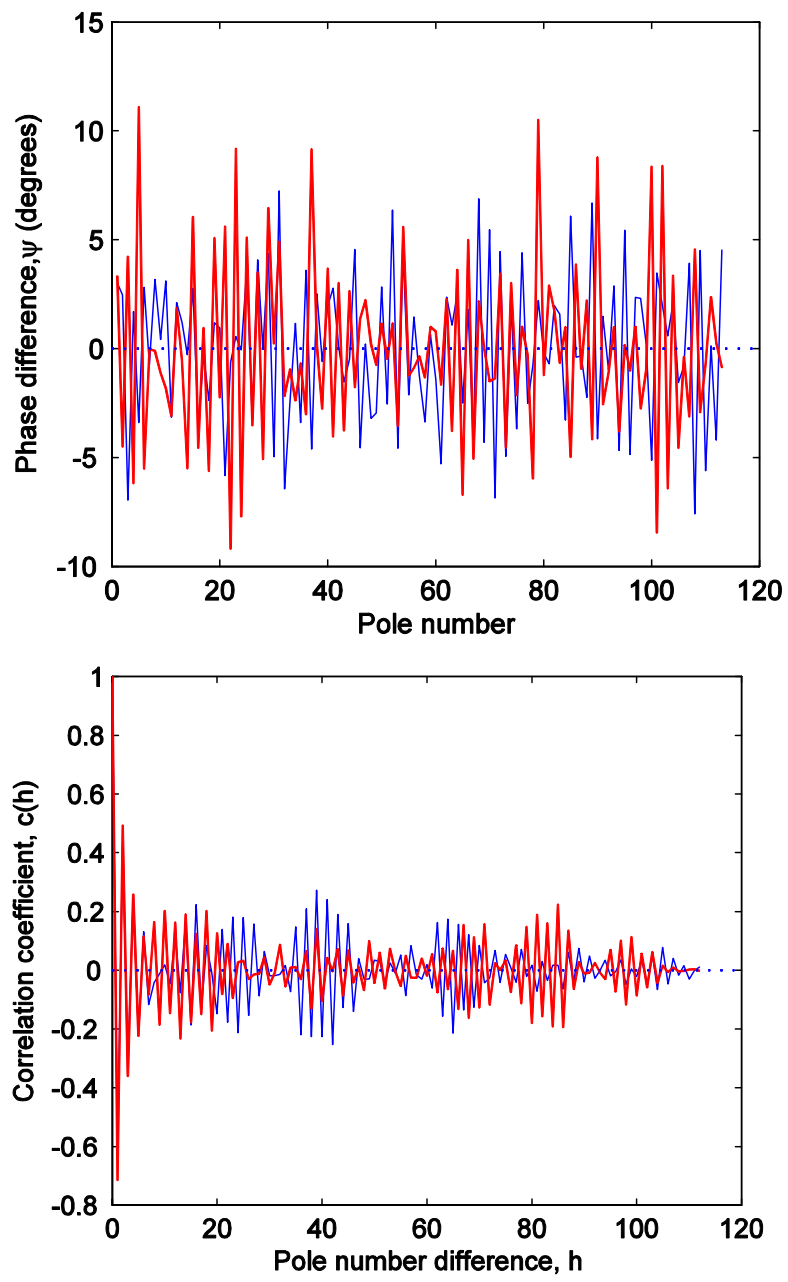

FIG. 7. Phase error differences (upper) and autocorrelation coefficients (lower) for the APS low (blue) and high (red) phase error devices.

The next-nearest pole difference is used rather than the phase difference between adjacent poles in order to eliminate the phase oscillation introduced by trajectory deviations. We can estimate the rms amplitude of the phase oscillations then from the following:

$$
\sigma_{\delta} \simeq \sqrt{\left\langle\varphi_{i+1}-\varphi_{i}\right\rangle^{2}-\sigma_{\psi}^{2}} / 2 .
$$

We can also determine the magnitude of the smooth phase variation, by fitting the phase distribution with a polynomial (an example of which is shown in Fig. 2) and then calculating its rms value, $\sigma_{\mathrm{sm}}$.

In order to determine if the phase errors are statistically consistent with what would be expected from a randomwalk process, we first remove the effect of the phase oscillation on the rms phase:

$$
\sigma_{\varphi, \mathrm{eff}} \simeq \sqrt{\sigma_{\varphi}^{2}-\sigma_{\delta}^{2}}
$$

and the maximum phase error, 

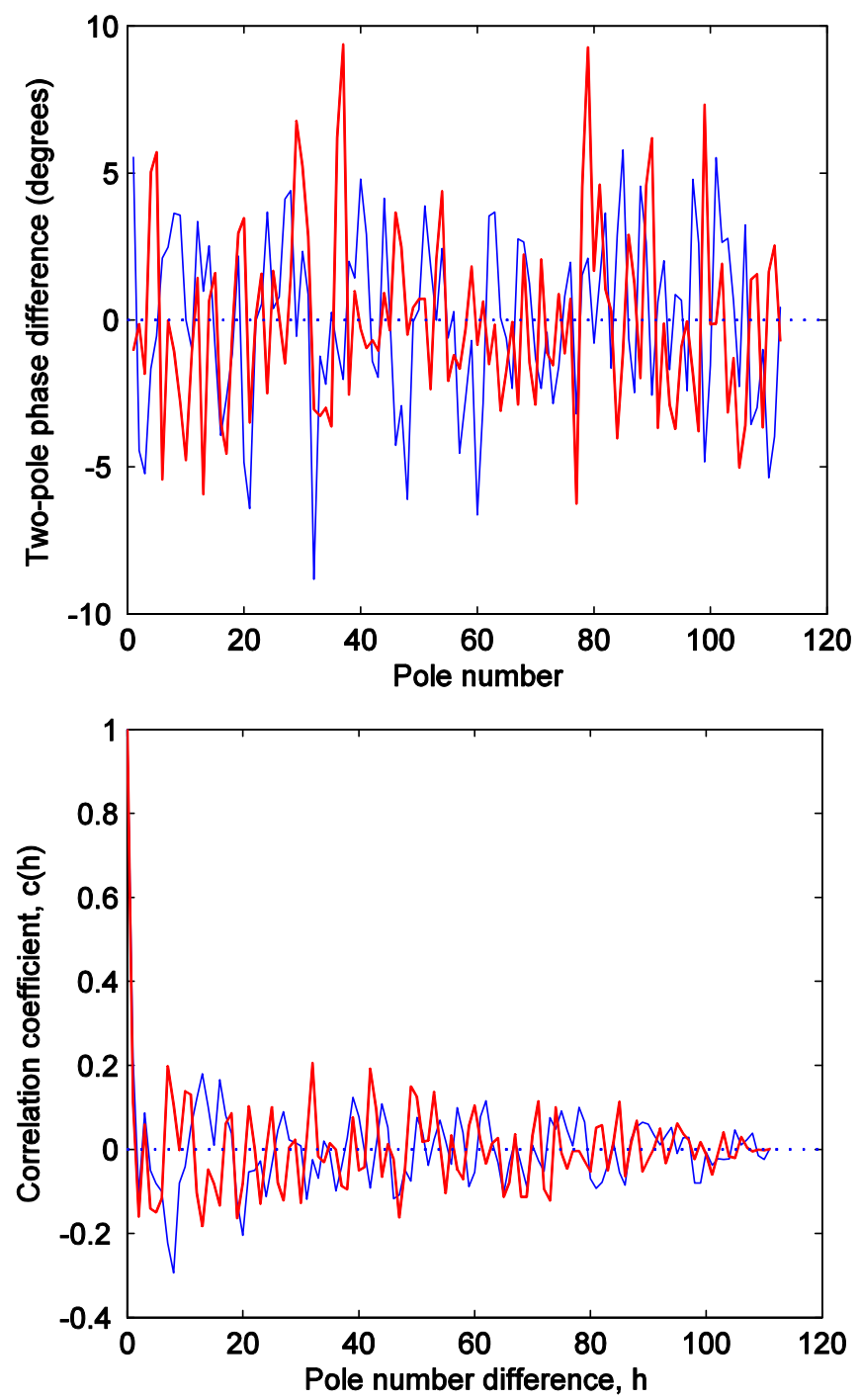

FIG. 8. Two-pole phase error differences (upper) and autocorrelation coefficients (lower) for the APS low (blue) and high (red) phase error devices.

$$
\varphi_{\max }=\max \left\{\left|\left(\varphi_{i+1}+\varphi_{i}\right) / 2\right|\right\}
$$

and then compare the values of these quantities with the local phase error, $\sigma_{\psi}$.

The results of these calculations for the ten cases that have been investigated are presented in Table I. It appears from these results that to a good approximation, the rms phase error is the quadratic sum of the constituent smooth, local random and phase oscillation terms:

$$
\sigma_{\varphi} \simeq \sqrt{\sigma_{\mathrm{sm}}^{2}+\sigma_{\psi}^{2}+\sigma_{\delta}^{2}}
$$

It is also evident that, for the large phase error cases, the majority of the error comes from the smoothly varying part. It is noticeable that the range of values of $\sigma_{\psi}$ is much less than that of $\sigma_{\varphi}$, also that for the two in-vacuum devices $\sigma_{\psi}$ does not differ very much between before and after shimming, whereas the ratio of the maximum phase error to $\sigma_{\psi}$ is much reduced. This is indeed what one would expect, namely, that the shimming reduces the overall excursion of the phase error, and rms value, with much less impact on the local errors present throughout the device.

We now return to the question of whether these phase errors are consistent with a random-walk process. In Fig. 9 we compare the results with what would be produced by a random-walk process based on normally distributed random local errors, $\psi_{i}$, as a function of the number of poles $n_{p}$. For each set of errors the data has been histogrammed to determine the most probable values (represented by the data points) while the error bars are defined by the points which enclose $90 \%$ of the data, i.e., $5 \%$ of the population are excluded on either side of the peak; similarly, the dotted lines contain $50 \%$ of the data, with $25 \%$ excluded on either side. The solid lines are the following: $\sigma_{\varphi} / \sigma_{\psi}=$ $\sqrt{n_{p}} / 1.5 \pi$ and $\varphi_{\max } / \sigma_{\psi}=\sqrt{n_{p}} / 2$, which are suggested by a numerical fit to the data. A dependence of the phase error on the square root of the number of poles was also observed by Bahrdt and Ivanyushkenov, when discussing the effect of random mechanical errors in superconducting undulators [17].

TABLE I. Characteristics of the phase errors for the ten different cases that have been studied. See text for details.

\begin{tabular}{lcccccccc}
\hline \hline Device & $n_{p}$ & $\sigma_{\varphi}(\mathrm{deg})$ & $\sigma_{\mathrm{sm}}(\mathrm{deg})$ & $\sigma_{\psi}(\mathrm{deg})$ & $\sigma_{\delta}(\mathrm{deg})$ & $\frac{\sigma_{\varphi, \text { eff }}}{\sigma_{\psi}}$ & $\frac{\varphi_{\max }}{\sigma_{\psi}}$ & Symbol \\
\hline APS low phase error & 114 & 3.7 & 2.5 & 2.1 & 1.3 & 1.6 & 3.2 & Red open circle \\
APS high phase error & 114 & 6.7 & 6.2 & 2.2 & 1.4 & 2.9 & 6.5 & Red solid circle \\
DLS U23 initial & 170 & 7.6 & 7.1 & 2.4 & 1.4 & 3.0 & 11.0 & Blue open circle \\
DLS U23 final & 170 & 3.0 & 2.0 & 2.0 & 1.2 & 1.4 & 3.3 & Blue solid circle \\
DLS U22 initial & 180 & 12.9 & 12.6 & 1.9 & 1.9 & 6.8 & 16.8 & Green open circle \\
DLS U22 final & 180 & 1.9 & 1.2 & 1.4 & 0.6 & 1.3 & 3.9 & Green solid circle \\
DLS HU64 & 64 & 6.8 & 6.0 & 2.8 & 2.7 & 2.2 & 5.7 & Pink open circle \\
DLS HU48 & 78 & 8.0 & 7.6 & 2.7 & 1.9 & 2.9 & 6.4 & Pink solid circle \\
LCLS\#1 & 216 & 3.5 & 2.5 & 1.6 & 1.0 & 2.1 & 5.4 & Black open circle \\
LCLS\#2 & 216 & 4.2 & 2.9 & 1.7 & 1.9 & 2.2 & 7.5 & Black solid circle \\
\hline \hline
\end{tabular}



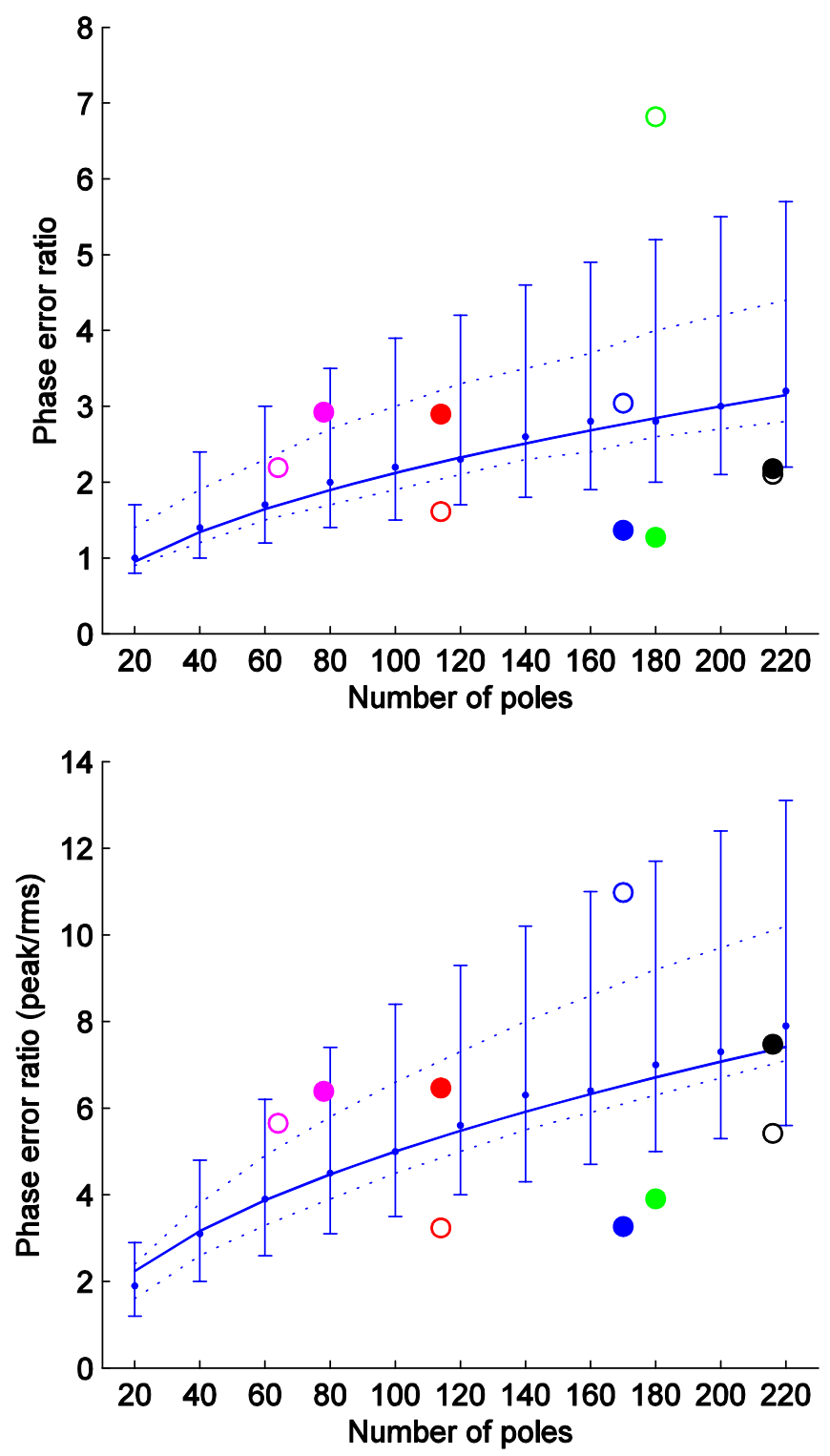

FIG. 9. Ratio between $\sigma_{\varphi \text {,eff }}$ and $\sigma_{\psi}$ (upper) and between $\varphi_{\max }$ and $\sigma_{\psi}$ (lower) as a function of the number of poles in an undulator for random sets of local phase errors $\left(\psi_{i}\right)$. Blue data points show the most probable values, error bars contain $90 \%$ of the population, and dotted lines $50 \%$ of the population. Data points correspond to the devices listed in Table I, with the corresponding symbol.

It can be seen that there is a large statistical spread in possible values of the two quantities which increases with the number of poles. Even so, of the ten examples studied, one shows significantly higher values than expected (one of the DLS in-vacuum devices before shimming) and four devices have lower values (the two DLS in-vacuum devices after shimming, the APS low phase error device and LCLS\#1). So, although a random-walk distribution of errors is generally a good description of errors in real devices, real devices exhibit an even greater range of variation of these two quantities than statistically expected.
In the case of the device with larger phase errors than expected from the underlying errors, the explanation is most probably a significant additional systematic phase variation. The four devices with statistically smaller phase errors are the ones with the overall smallest rms phase errors, and so these devices were most probably shimmed to the greatest extent: the more shimming is applied the more the resulting errors approach statistically independent phase errors. As regards the amount of phase oscillation, in all cases $\sigma_{\delta}$ is significantly larger than would result from random local errors, and so are attributed to trajectory deviations, which typically increase the overall rms phase error by $5 \%-10 \%$.

We proceed now to investigate the effect of the phase error distribution on the spectral performance.

\section{SPECTRAL PERFORMANCE}

\section{A. Spectral calculation}

To reduce computational time so that a large number of different cases can be calculated, use is made of the approximation of the radiation spectrum (i.e., the brightness, photon flux per unit relative bandwidth, and unit solid angle) as the emission from a series of dipole magnets with appropriate phase factors, as introduced in Ref. [5]:

$\frac{d^{2} \Phi}{d \omega / \omega d \Omega}=\frac{3 \alpha}{4 \pi^{2}} \frac{I}{e} \gamma^{2}\left(\frac{\varepsilon}{\varepsilon_{c}}\right)^{2}\left[1+\left(\gamma \theta_{y}\right)^{2}\right]^{2}\left[\left|A_{x}\right|^{2}+\left|A_{y}\right|^{2}\right]$

where $\alpha$ is the fine structure constant, $\varepsilon$ is the photon energy, $\varepsilon_{c}$ is the critical photon energy, $I$ is the beam current, $\theta_{y}$ is the vertical emission angle, and $A_{x}$ and $A_{y}$ are the complex radiation amplitudes. In the current context where the vertical angle is small, we can neglect the vertically polarized radiation component and write

$$
\begin{aligned}
A_{x}= & K_{2 / 3}(\xi)\left(e^{j \varphi_{1}} e^{j \delta_{1}}+e^{j \varphi_{2}} e^{-j \delta_{1}} e^{j \pi \delta}+e^{j \varphi_{3}} e^{j \delta_{1}} e^{j 2 \pi \delta}\right. \\
& \left.+e^{j \varphi_{4}} e^{-j \delta_{1}} e^{j 3 \pi \delta}+\cdots\right),
\end{aligned}
$$

where $\xi=y\left[1+\left(\gamma \theta_{y}\right)^{2}\right]^{3 / 2} / 2$. In the above $\varphi_{i}$ are the phase errors at the poles, $\delta$ is the regular change in phase per pole,

$$
\delta=\frac{X+Y^{2}}{N}
$$

which is introduced by frequency detuning with respect to the on-axis value,

$$
X=n N\left(\Delta \omega / \omega_{n, 0}\right),
$$

or from observing the radiation off axis,

$$
Y^{2}=n N \frac{\gamma^{2} \theta^{2}}{1+K^{2} / 2},
$$


where $\theta^{2}=\theta_{x}^{2}+\theta_{y}^{2}$ and $N$ is the number of periods. With this definition, $X=1$ when $\Delta \omega / \omega_{n, 0}=1 / n N$ and $Y^{2}=1$ when $\theta^{2}=\left(1+K^{2} / 2\right) / n N \gamma^{2}=2 \lambda / L$, with $\lambda$ the radiation wavelength and $L$ the undulator length. Under either of
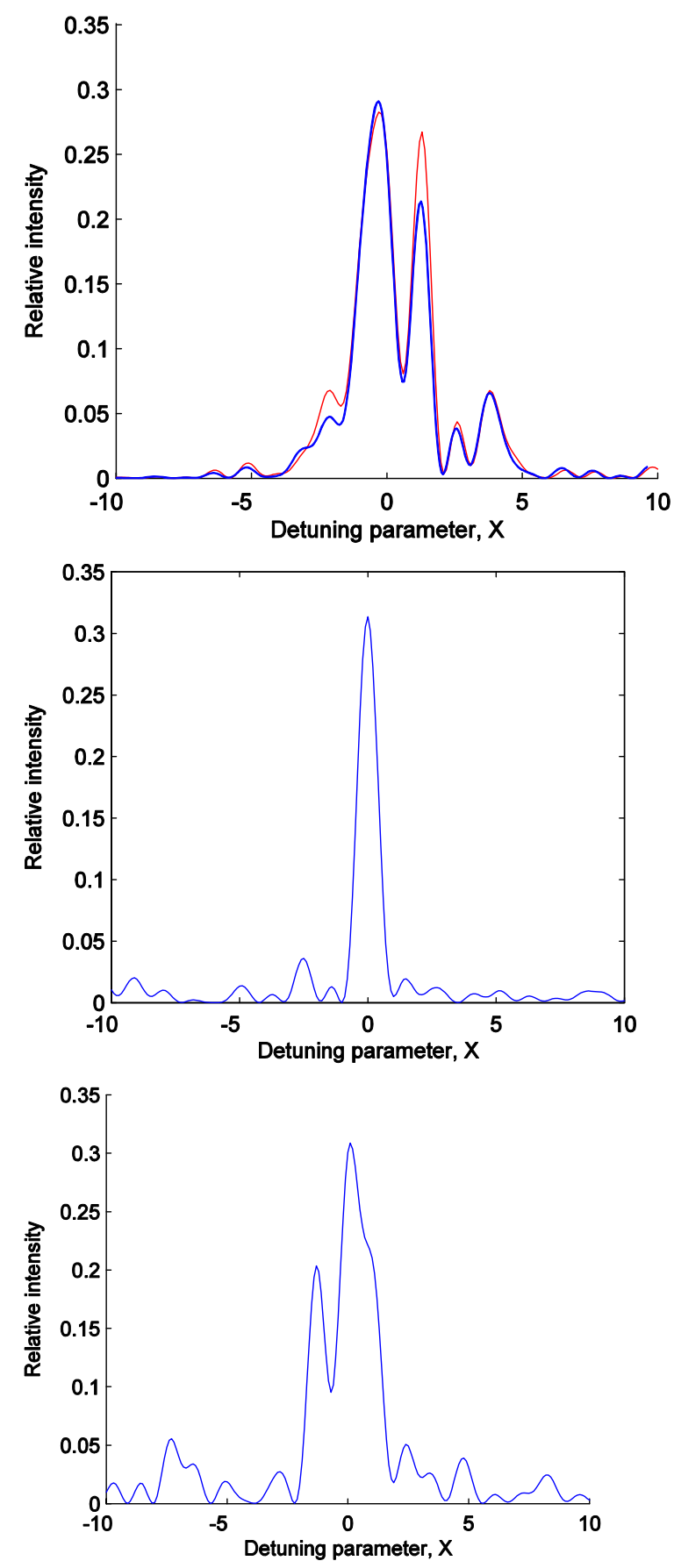

FIG. 10. On-axis, single-electron brightness for the 9th harmonic, for different phase error distributions each with the same rms value of $\sigma_{\varphi}=6.7^{\circ}$; upper-the APS high phase error device; blue curve, using the code UR [18]; red curve, using the "dipole series model"; middle-the same set of random errors as Fig. 1; lower-the same sets of random-walk errors as Fig. 2. Intensities are normalized to the peak value for the ideal case, without errors. these conditions there is a cancellation over the $N$ periods of the device and, hence, zero intensity (in the absence of phase errors). The remaining phase term, $\delta_{1}$, is the difference in phase between positive and negative poles which results from the horizontal angle of emission [5]:

$$
\delta_{1}=\frac{\omega}{\omega_{1, \theta}}\left(k z+\frac{3 K^{2} \sin 2 k z}{4\left(1+K^{2} / 2+\gamma^{2} \theta^{2}\right)}\right)
$$

where $k z=-\sin ^{-1}(\alpha)$ and $\alpha=\gamma \theta_{x} / K$.

In the case of radiation emitted on axis, Eq. (4) takes on a very simple form:
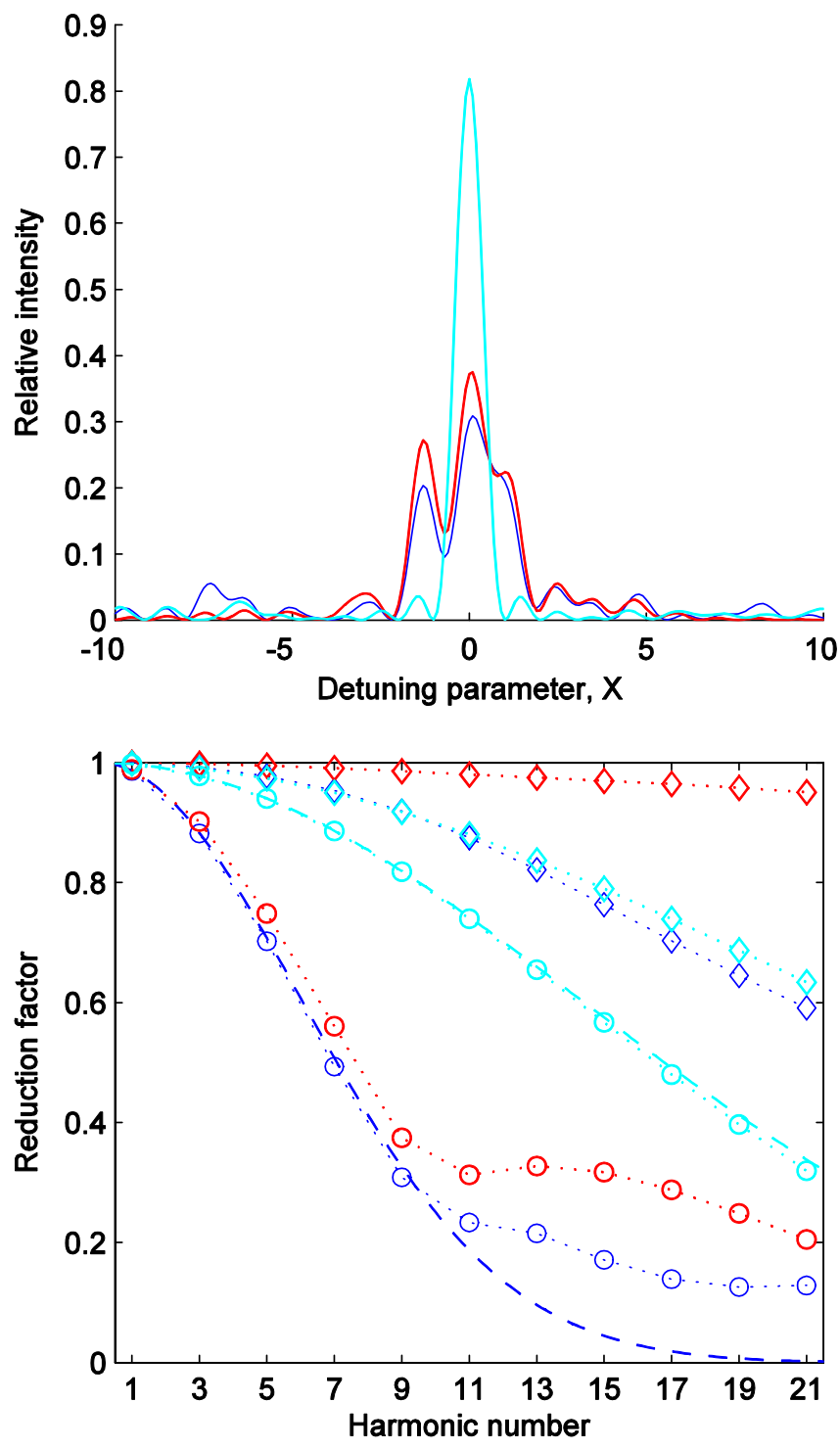

FIG. 11. On-axis single-electron brightness spectra for the 9th harmonic (upper) and reduction factors (lower) for peak (circles) and integrated (diamonds) brightness as a function of harmonic number for the phase errors of Fig. 2. Colors in both plots refer to those of Fig. 2. The dashed lines are Eq. (1) with $\sigma_{\varphi}=6.7^{\circ}$ (blue) and $2.8^{\circ}$ (cyan). 


$$
\begin{gathered}
\frac{d^{2} \Phi}{d \omega / \omega d \Omega} \sim\left|A_{x}\right|^{2}, \\
A_{x}=e^{j \varphi_{1}}+e^{j \varphi_{2}} e^{j \pi \delta}+e^{j \varphi_{3}} e^{j 2 \pi \delta}+\cdots, \quad \delta=\frac{X}{N} .
\end{gathered}
$$

The on-axis, single-electron brightness spectrum is thus very easily calculated from a set of phase error and so we will start the analysis of the effect of phase errors on the radiation emission using this approximation, i.e., with zero electron beam emittance and energy spread and with zero angular acceptance.

\section{B. Effect of phase errors on the single-electron emission spectrum}

Figure 10 (upper) shows the brightness spectrum for the high phase error APS device on the 9th harmonic. First, it can be seen that the simple dipole series model (red curve) agrees very well with the result of a detailed calculation based on the magnetic field distribution using the code UR [18] (blue curve). Figure 10 (middle) shows the result for the same set of simple random phase errors as Fig. 1. The
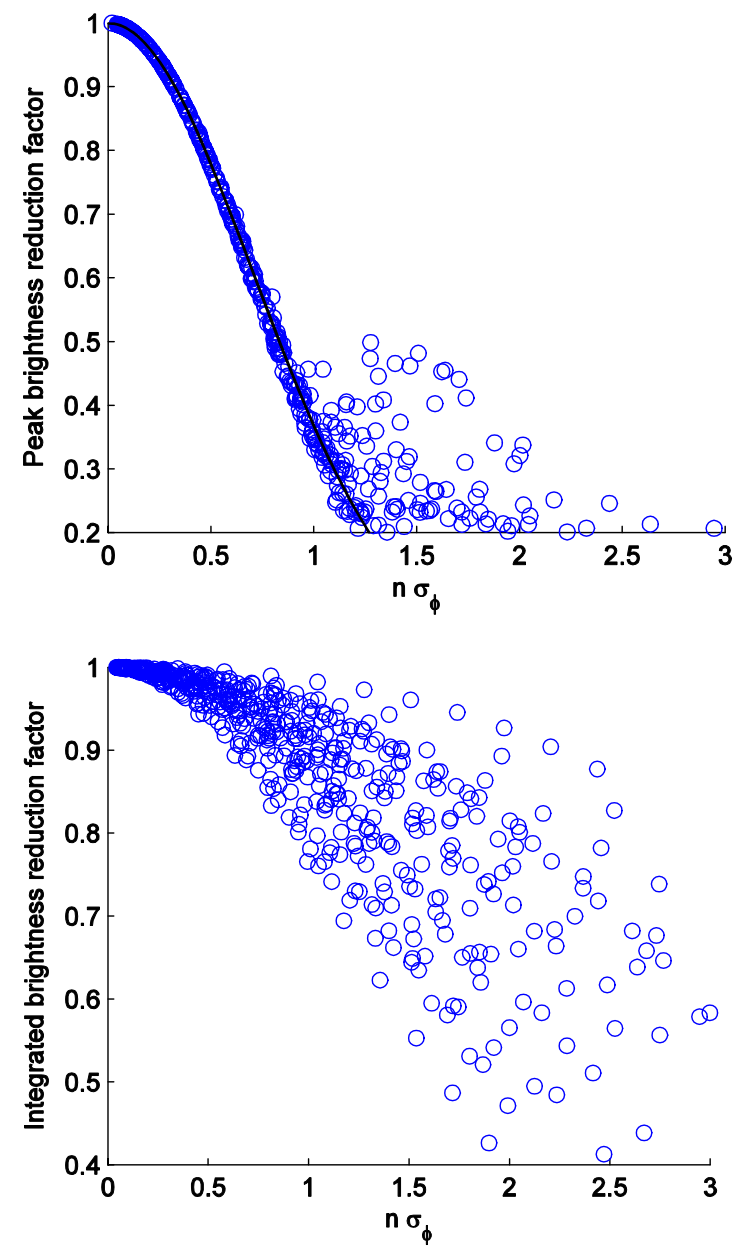

peak intensity is the same, however the spectrum is much narrower, in fact of the same width as the ideal case with no errors (with intensity zeros at $X= \pm 1$ and little intensity outside that range). Figure 10 (lower), on the other hand, is for the set of random-walk errors from Fig. 2. While the peak value is again very similar, the spectrum is now broader, as in the upper plot. This immediately suggests a reason for the observed reduction of the influence of phase errors in practical cases: when integrating over energy spread, emittance, and collection angle, there is effectively an integration over the brightness spectrum, and hence the reduction in peak brightness is compensated by the broadening of the spectrum. In the following we will use the easily calculated "integrated brightness," i.e., the integration over the single-electron, on-axis brightness spectrum as a guide to the behavior of the true integrated flux, and later show the validity of this assumption.

We first investigate the relative effects of the "smooth" and "random" components of the typical set of phase errors shown in Fig. 2. Figure 11 shows the 9th harmonic spectra for the two components; the smooth part of the
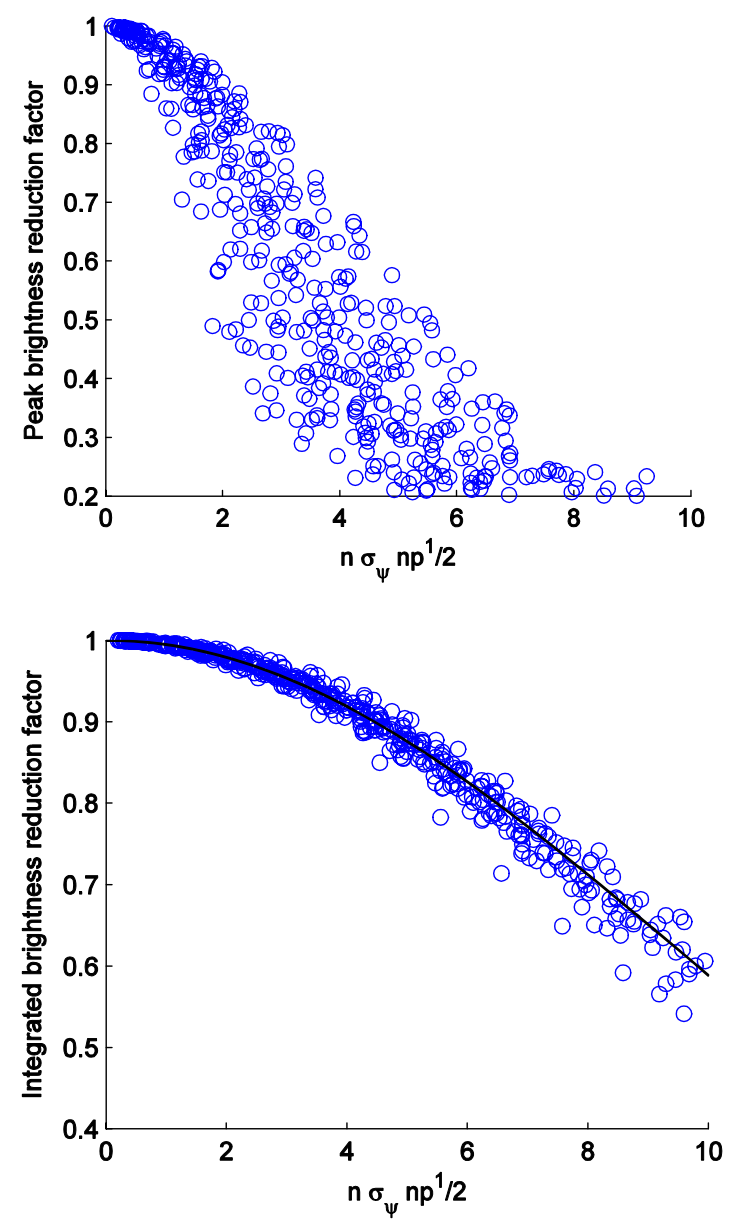

FIG. 12. Reduction factors in single-electron peak (upper plots) and integrated (lower plots) brightness, as functions of rms phase error (left-hand plots) and rms local phase error (right-hand plots) in radians, for random-walk errors with a range of values of $\sigma_{\psi}$ and $n_{p}$, and odd harmonics 1-21. The solid lines are upper-left-Eq. (1); lower-right-Eq. (6). 
phase errors (red curve) produces a similar shaped spectrum to the complete set of errors (blue curve) but with larger integral, whereas the random part on its own produces a higher peak value, but narrower spectrum. The lower plot shows the reduction factors for peak and integrated brightness (where the reduction factor is the ratio between the quantity with and without phase errors) for the three cases. It is interesting to relate these also to the error statistics: the full set of errors has an rms phase of $6.7^{\circ}$, and rms local phase error of $2.5^{\circ}$. The smooth part of the error has a similar rms phase of $6.1^{\circ}$, but much smaller local errors, $1.3^{\circ}$; the random part, on the other hand, shows a much lower rms phase of $2.8^{\circ}$, and similar local errors to the full set, $2.4^{\circ}$. Thus, the smooth part of the random-walk errors (red curves), which makes up the major part of the overall rms phase, determines the peak brightness, while the random component which relates to the local phase error (cyan curves) determines the integrated brightness. We can therefore begin to appreciate why certain phase error distributions, namely, those dominated by smooth/systematic errors, result in less reduction in the integrated flux than in the peak brightness.

We now look in more detail at how the peak brightness and integrated brightness depend on the random-walk phase error statistics. For each data point in Fig. 12, a value of rms phase difference was selected at random in the range $1^{\circ}-3^{\circ}$ and number of poles in the range 50-250. Random-walk phase errors were generated and then peak and integrated brightness calculated. The plots show how the reduction factors due to phase errors for these two quantities depend on rms phase error $\sigma_{\varphi}$, and also on the new parameter introduced in Sec. II B, the rms local phase error, $\sigma_{\psi}$. The results confirm again the observation that the integrated brightness is much less reduced by phase errors than the peak brightness. The peak brightness shows again the usual dependence on rms phase according to Eq. (1). For the integrated brightness a series of simulations revealed a dependence on the square root of the number of poles (as observed earlier in relation to Fig. 9), i.e., on the variable $n \sigma_{\psi} n_{p}^{1 / 2}$. The fitted curve shows that the reduction factor for the integrated brightness depends as follows:

$$
R=\exp \left(-c n^{2} \sigma_{\psi}^{2} n_{p}\right)
$$

where $c$ is a constant; in the case of Fig. 12, lower right, a numerical fit gives $c \simeq 1 / 188.6$.

Finally, we examine the effect of systematic phase errors and phase oscillations on the radiation emission. Figure 13 (upper) shows the spectrum of the 9th harmonic for the systematic errors of Fig. 3 and it is clear that, while there is a significant reduction in the peak brightness, the spectrum is even broader than for random errors (Fig. 10). This is confirmed in the lower plot which shows the reduction factors for peak and integrated brightness for these cases as a function of harmonic number. There is a clear
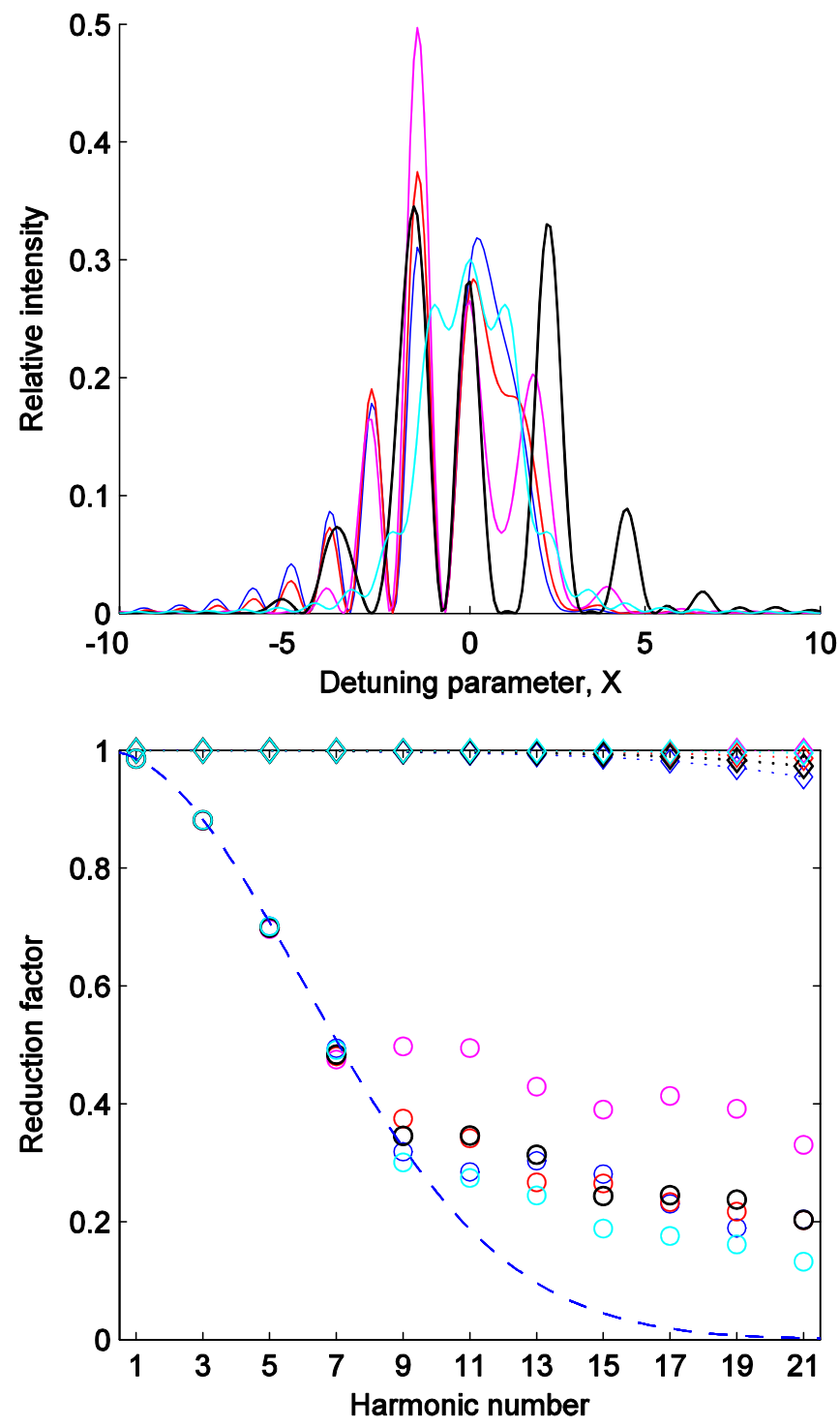

FIG. 13. On-axis single-electron brightness spectra for the 9th harmonic (upper) and reduction factors (lower) for peak (circles) and integrated (diamonds) brightness for the four cases of systematic phase errors shown in Fig. 3, using the corresponding colors. The dashed line is Eq. (1) with the relevant rms phase error, $\sigma_{\varphi}=6.7^{\circ}$.

similarity to the smooth part of the random-walk errors in Fig. 11. While the reduction in peak brightness again follows Eq. (1), at least down to the $50 \%$ level, the integrated intensity shows a much reduced influence of phase errors. We can conclude therefore that smoothly varying phase errors, whether arising from the random-walk process or from systematic effects, produce a similar effect on the radiation properties.

Phase errors due to trajectory angle errors, on the other hand, have a markedly different effect as shown in Fig. 14. Here the reduction in peak brightness, which again follows Eq. (1), is not compensated by any broadening of the onaxis spectrum and so the reduction in integrated brightness 

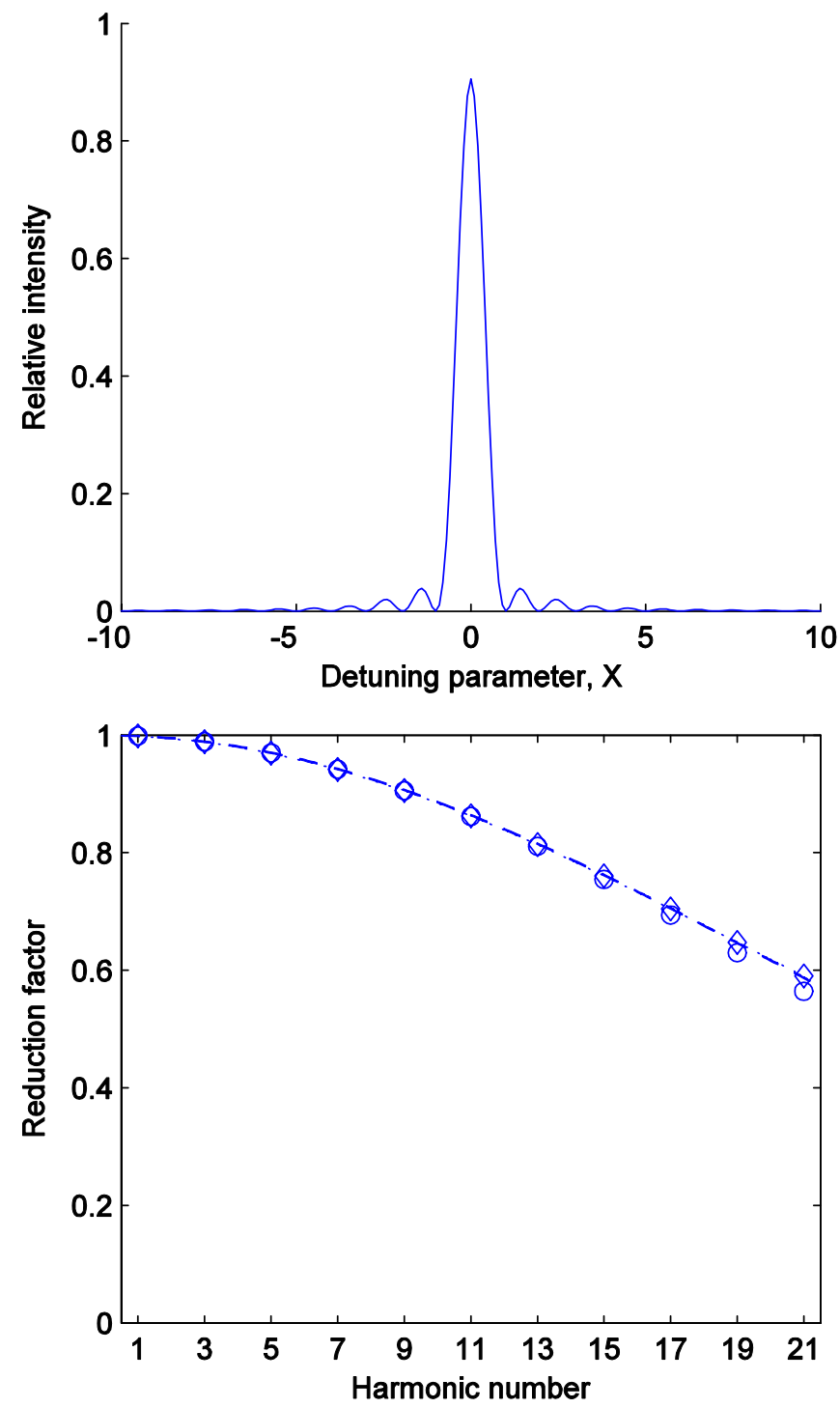

FIG. 14. On-axis single-electron brightness spectrum for the 9th harmonic (upper) and reduction factors (lower) for peak (circles) and integrated (diamonds) brightness as a function of harmonic number (lower) for the phase errors of Fig. 5. The dashed line is Eq. (1) with the relevant phase error $\sigma_{\varphi}=2^{\circ}$.

is the same as that of the peak brightness. The situation in practice however is not as dramatic as it might appear from this result. When integrated over a range of collection angles, and including the effect of energy spread, the apparent reduction in intensity can be recovered.

\section{Effect of phase errors in real devices on the single-electron emission spectrum}

Having examined the general effect of different types of phase error on the radiation properties, we now proceed to investigate further the previous selection of real devices. Figure 15 shows the reduction in peak and integrated brightness for the two APS devices, as a function of

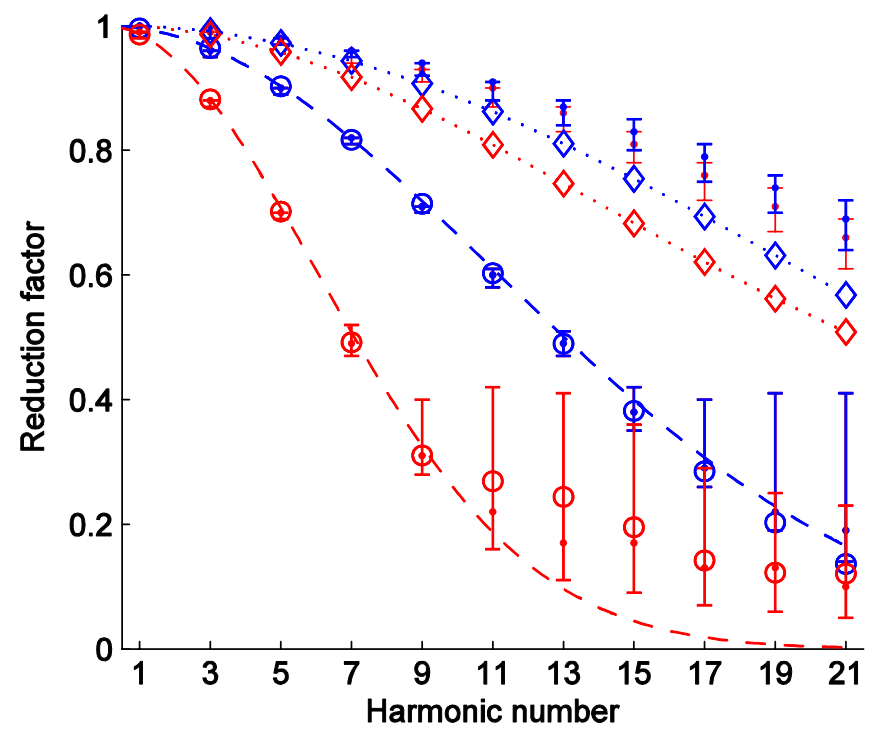

FIG. 15. Reduction factors for peak (circles) and integrated (diamonds) single-electron brightness for the low (blue) and high (red) phase error APS devices, as functions of harmonic number. The dashed lines are Eq. (1), with the appropriate rms phase errors for the two devices. Error bars indicate the range of results expected from a random-walk phase model, using the appropriate $\sigma_{\varphi}$ for the peak brightness and $\sigma_{\psi}$ for the integrated brightness calculations from Table I; points give the most probable value of the distribution and error bars show the range that includes $90 \%$ of the population (i.e., exclude $5 \%$ of the data on either side of the peak).

harmonic number. Again it can be seen that the peak brightness closely follows Eq. (1), except at very low intensity ratios; however, the integrated intensity shows much less reduction with increasing harmonic number, similar to the results in the previous section. Figure 15 also shows what would be expected for random-walk errors with the corresponding values of $\sigma_{\varphi}$ and $\sigma_{\psi}$ for the peak and integrated brightness, respectively. While the reduction factor for peak brightness is consistent with the random-walk model [and up to a point also Eq. (1)], there is some discrepancy for the integrated brightness, the real devices giving a somewhat larger reduction factor.

We proceed to examine the other devices listed in Table I to see if their performance is also consistent with randomwalk errors. Figure 16 shows the reduction factors in peak and integrated brightness for the various devices, plotted in the same way as in Fig. 12 against rms phase and local phase errors. The upper plots show again that peak brightness depends on the phase and follows Eq. (1). The lower plots, on the other hand, show that integrated brightness correlates much better with the rms local phase error, as in Fig. 12, but with some discrepancy with respect to the fitted curve, Eq. (6). By inspection it appears that the deviation from the model correlates with the size of the phase oscillations, $\sigma_{\delta}$, and indeed this is confirmed by Fig. 17, 

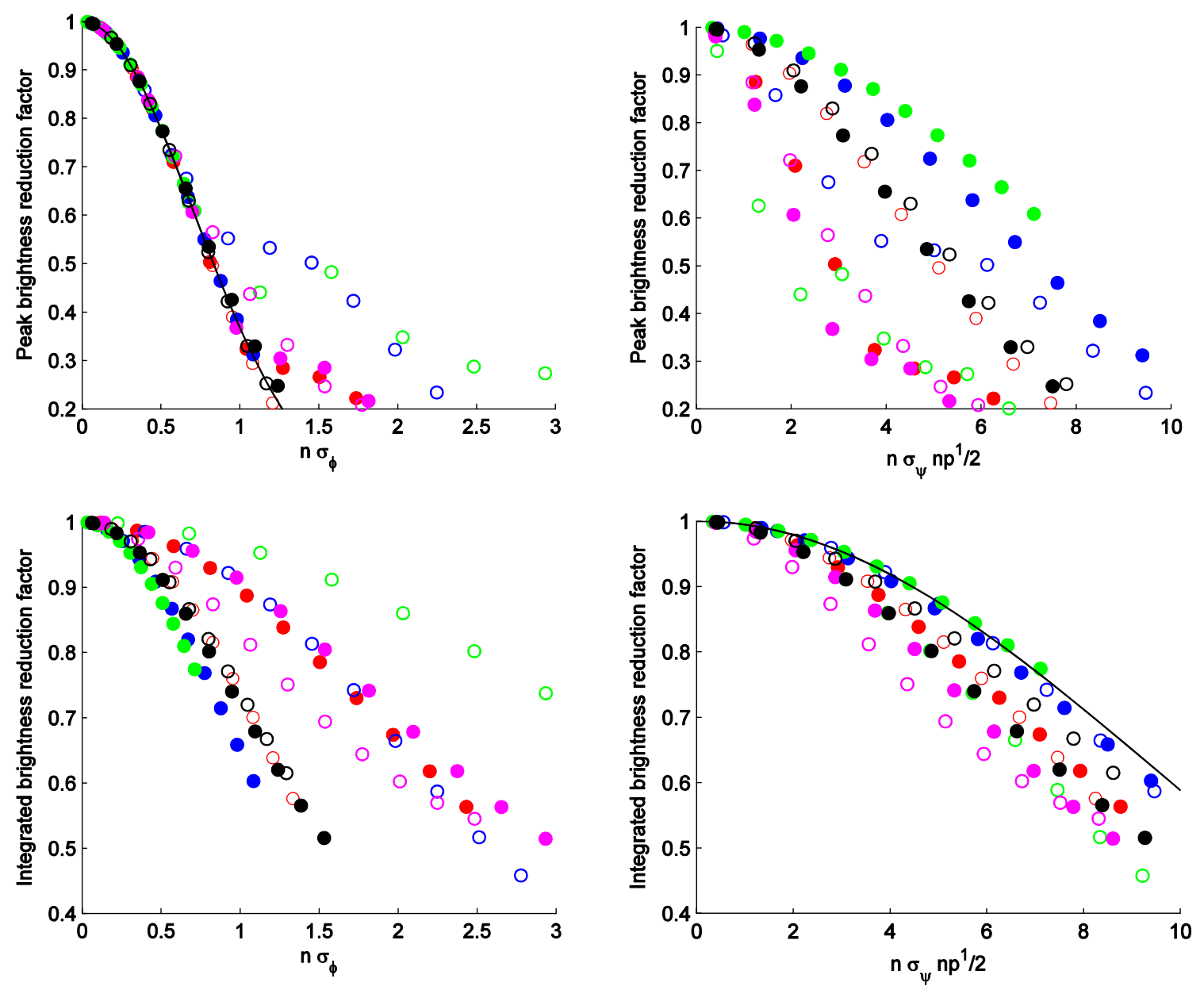

FIG. 16. Reduction factors in single-electron peak (upper plots) and integrated (lower plots) brightness, as functions of rms phase error (left-hand plots) and rms local phase error (right-hand plots) in radians, for the ten devices of Table I with corresponding symbols. Solid lines: upper-left plot—Eq. (1); lower-right plot-Eq. (6).

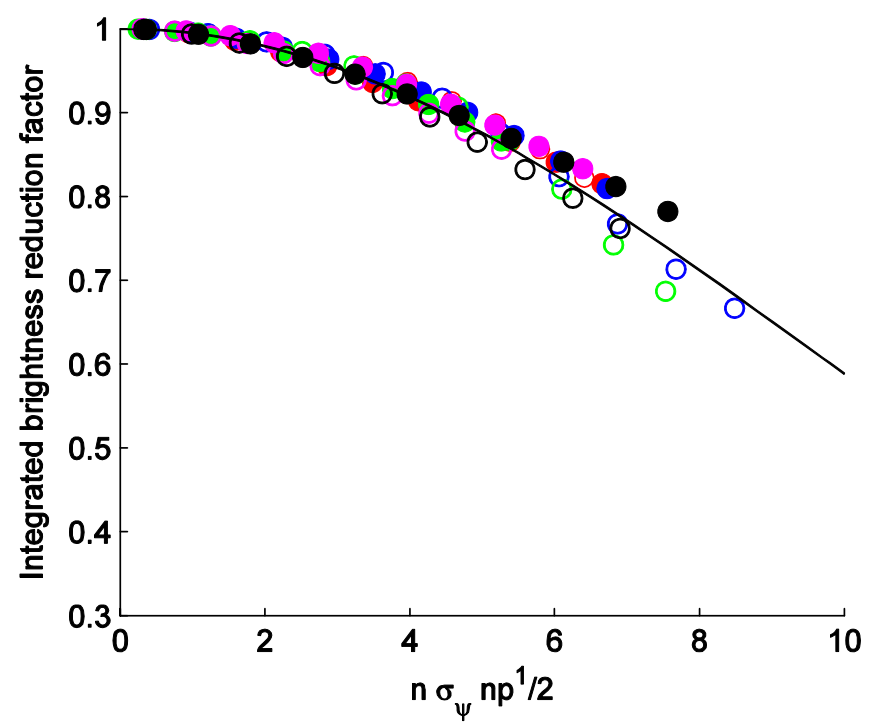

FIG. 17. As Fig. 16 (lower right), after removing the effect of phase oscillations. in which the phase errors are effectively removed by averaging the phase between adjacent poles.

\section{Effect of phase errors in real devices including electron beam parameters and pinhole aperture}

We now extend the analysis to include the effects of electron beam emittance, energy spread, and finite collection angles on the emission spectra. We first compare the results obtained with the dipole series model, Eq. (4), with the results of accurate calculations carried out with the code UR [18]. Figure 18 compares the flux integrated over a pinhole aperture for the 15th harmonic, and Fig. 19 compares the reduction factors for the integrated (pinhole) flux and peak on-axis brightness, in the case of the APS "high phase error" device. It can be seen that the results obtained with the dipole series model are in good agreement with the code UR, and also that the simple method of estimating the reduction factor of integrated flux used previously gives the correct general trend of the falloff with increasing harmonic number. 

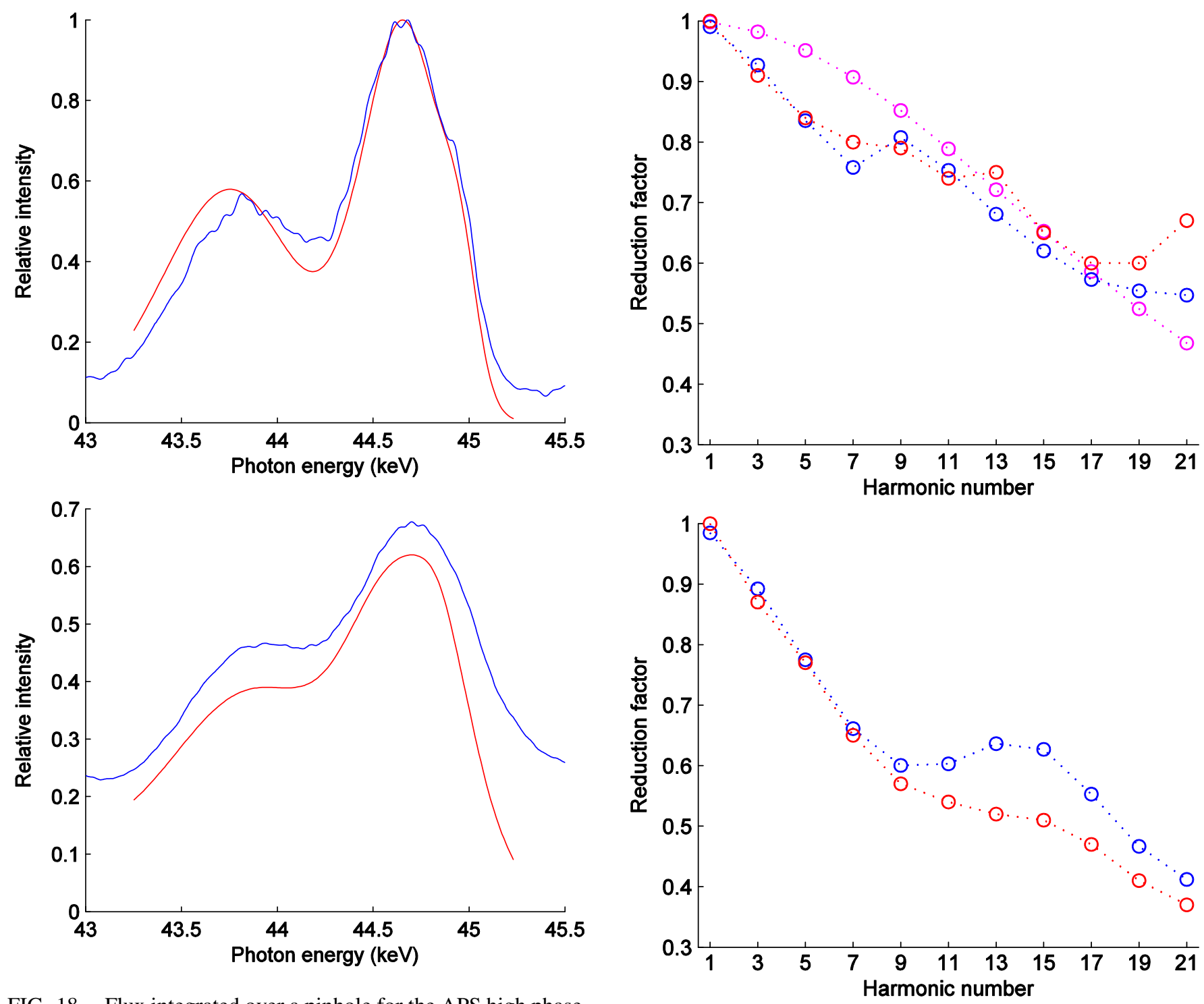

FIG. 18. Flux integrated over a pinhole for the APS high phase error device in the 15th harmonic, including emittance and energy spread. Upper-ideal case without phase errors; lower-with phase errors. Blue curves-calculated with the code UR [18]; red curves - using the current model. Parameters are the same as in Ref. [13]: energy $=7 \mathrm{GeV}$, rms horizontal/vertical electron beam divergences $=11.1 / 4.9 \mu \mathrm{rad}, \quad$ energy $\operatorname{spread}=9.6 \times$ $10^{-4}$, pinhole size $=2.5 \mathrm{~mm} \times 1 \mathrm{~mm}$ at $30 \mathrm{~m}$.

Having confirmed the validity of the dipole series model also in the case of off-axis emission, Fig. 20 now shows the reduction factors for the on-axis brightness and the integrated flux, including emittance and energy spread, for the devices listed in Table I, plotted as in Fig. 16 as functions of rms phase and local phase errors. Here, arbitrarily, the same APS beam and pinhole parameters have been used also for the LCLS devices, while parameters appropriate for Diamond have been used for the those devices. For the peak brightness, there is a good agreement with the more simple calculation of Fig. 16, and also Eq. (1), only for reduction factors larger than about 0.8 ; below this level

FIG. 19. Reduction factors in integrated flux (upper) and onaxis brightness (lower), calculated with UR [18] (red curves) and the dipole series model (blue curves), for the APS high phase error device. The magenta curve in the upper plot is the simplified estimate for the reduction factor in integrated flux based on the on-axis brightness spectrum. APS parameters are as in Fig. 18.

there is considerably more scatter in the reduction factors, and in all cases less reduction in brightness than for the previous result. This is to be expected as the inclusion of emittance and energy spread allows the broadening of the brightness spectrum to compensate for the reduction in peak brightness.

For the integrated flux there is a good agreement between the two methods of calculation, which provides further justification for the use of the simple calculation as a guide to performance, and also the use of the rms local phase as a "figure of merit" for the integrated flux. It should be noted, however, that this agreement depends 

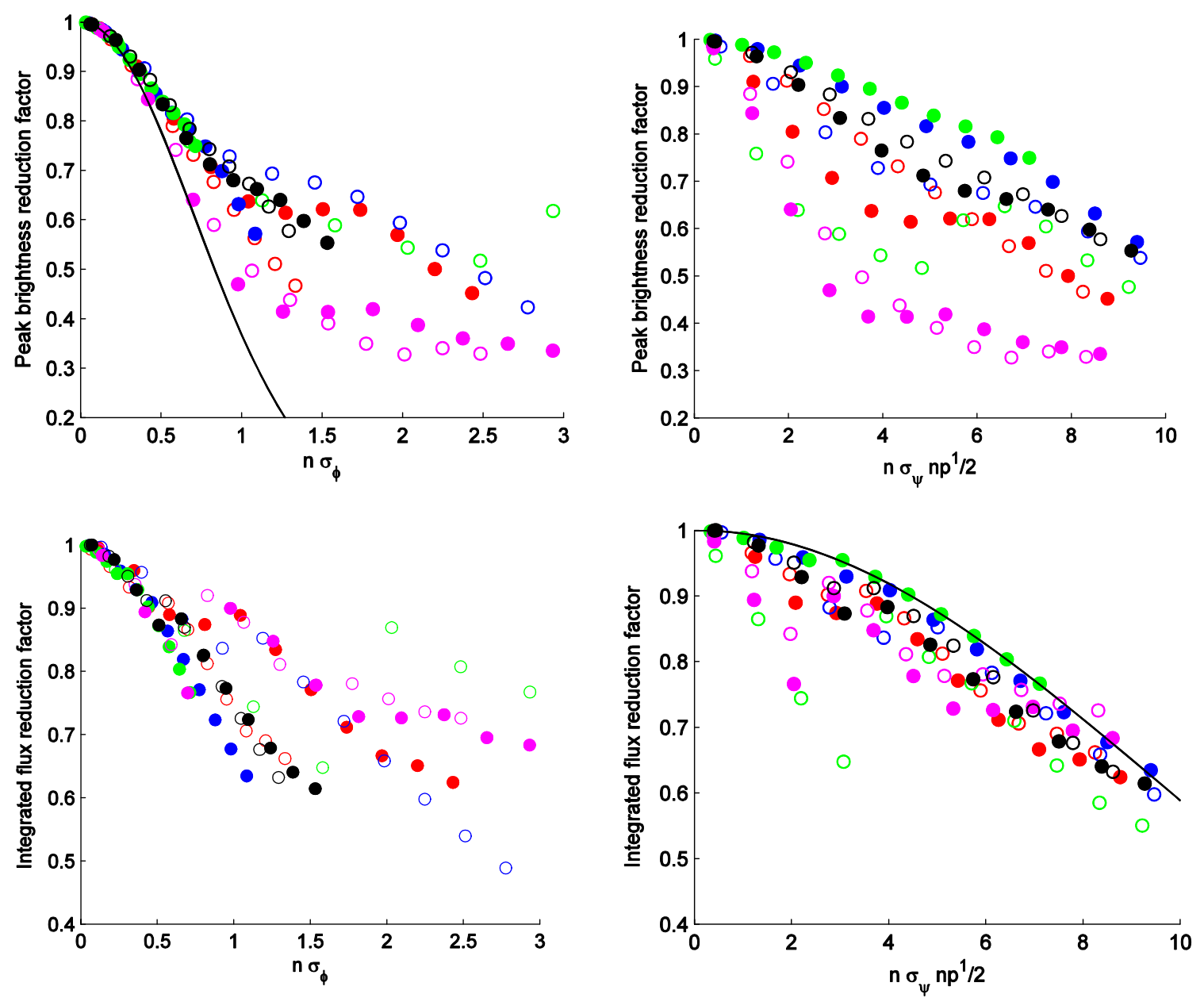

FIG. 20. Reduction factors in peak brightness (upper plots) and integrated flux (lower plots) as functions of rms phase error (lefthand plots) and rms local phase error (right-hand plots) in radians, for the ten devices of Table I with corresponding symbols, including the effects of emittance and energy spread, and integration over an aperture. Solid lines: upper-left plot-Eq. (1); lower-right plotEq. (6). Devices 1, 2, 9, and 10 use APS parameters as in Fig. 18, other devices: energy $=3 \mathrm{GeV}$, rms horizontal/vertical electron beam divergences $=24.2 / 4.2 \mu \mathrm{rad}$, energy spread $=9.6 \times 10^{-4}$. Pinhole sizes are set to collect approximately $95 \%$ of the peak flux in the fundamental.

very much on the size of the aperture. If the aperture is reduced then the reduction factors decrease for the larger rms phase error cases, increasing the scatter of points when plotted against the local phase error, but conversely reducing the scatter when plotted against the phase error, tending therefore (not surprisingly) towards the results for the peak brightness.

\section{THE EFFECT OF PHASE ERRORS IN FUTURE LOWER EMITTANCE RINGS}

The question of whether phase errors become more critical for lower emittance storage rings is a very topical one, with machines with sub-nm emittance such as MAX-IV being built [19] and "ultimate storage rings" with emittances down to the few 10's of pm range under active consideration [20]. Repeating the calculations of the section above with lower emittance, for example $1 / 10$ th of the nominal beam divergences, and (for the integrated flux) correspondingly reduced aperture, shows in fact very little difference. For the integrated flux this is not surprising; if the aperture is set to collect a high fraction of the flux, the reduced emittance serves only to reduce the extent of that aperture.

For the peak brightness there is a more noticeable effect, with a general increase in the effect of phase errors, in the limit of large phase errors. The limit of zero emittance becomes dominated by the energy spread. Figure 21 shows the reduction factor for that case. It should be noted that the energy spread used in these calculations $(\sim 0.1 \%)$ is also typical for future low emittance and "ultimate" storage rings, so this result is applicable to those cases.

Based on the results that have been presented, we can now reassess what phase errors are acceptable, assuming arbitrarily a requirement for a reduction factor no smaller than 0.75 due to phase errors, for operation up to the 15 th 


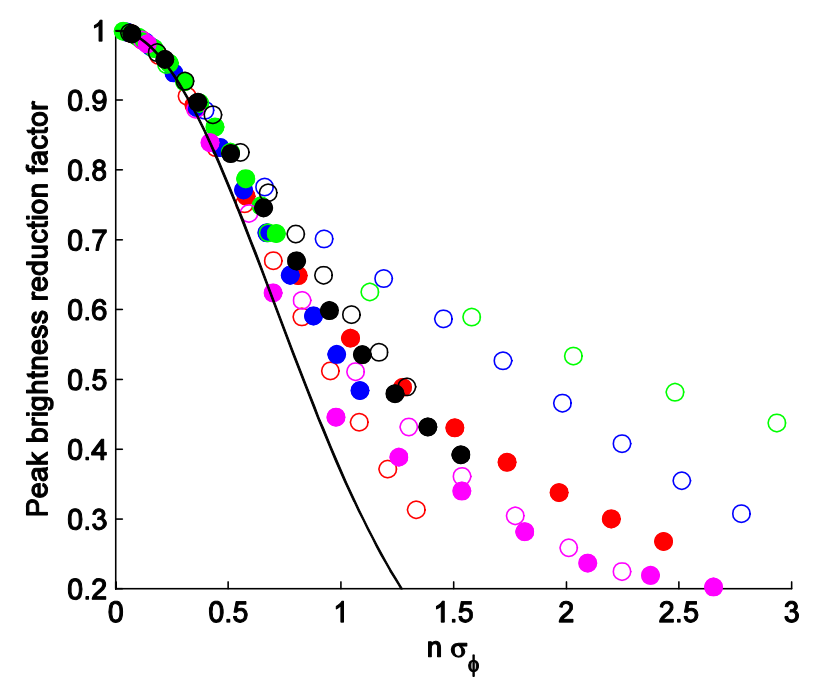

FIG. 21. As Fig. 20 (upper left), with zero electron beam emittance.

harmonic. Equation (1), which as shown is always pessimistic, would predict that the rms phase error should be less than $2^{\circ}$. If peak brightness is the figure of merit for the performance of the device in question, Fig. 20 (upper left) and Fig. 21 show little difference in this range, and would suggest that a phase error of $<2.3^{\circ}$ is needed $\left(n \sigma_{\varphi} \leq 0.6\right)$. If however integrated flux is the figure of merit, then there is considerably more variability in the performance of typical devices. According to Fig. 20 (lower left), "to be on the safe side" an rms phase of $<2.9^{\circ}$ would be needed $\left(n \sigma_{\varphi} \leq 0.75\right)$, although devices with phase errors 2-3 times larger could in principle satisfy the requirement, depending on the precise distribution of errors. The simulated device of Ref. [14] with a phase error of $6^{\circ}$ and a reduction factor for the integrated flux of greater than 0.75 up to the 15 th harmonic (i.e. $n \sigma_{\varphi}=1.57$ ) is one such particular case, consistent with the spread of points in Fig. 20, but clearly this cannot be taken as a general result. A better way of stating the requirement which involves less statistical uncertainty is to use the newly introduced rms local phase error. The requirement then becomes $n \sigma_{\psi} n_{p}^{1 / 2} \leq 5$ which for $n=15$ gives $\sigma_{\psi}<1.9^{\circ}$ for a device with 100 poles, and $\sigma_{\psi}<1.4^{\circ}$ for 200 poles.

\section{SUMMARY AND CONCLUSION}

The cumulative buildup of phase error from local random field errors in an insertion device follows a random-walk process. A new parameter, the rms local phase error, has been introduced as a measure of these underlying phase errors, which complements the usual rms phase parameter which describes the overall variation of (cumulative) phase.

The random-walk process results in both a smooth variation in phase and a random variation about that. An additional smooth variation may also arise from a systematic slow variation of gap (or other parameter), while an oscillation of the phase between neighboring poles can result from trajectory angle errors. To a good approximation, the rms phase error is the quadratic sum of the smooth, local random, and oscillatory terms.

In general, it does not appear possible to distinguish whether a particular smooth variation in phase comes from the random-walk process or from systematic effects, but since they have similar effects on the radiation properties, this distinction is not generally a concern. If however the phase error is large compared to the local phase error, to the extent that it is significantly outside the range of statistical probability, this would point to a systematic variation in gap or some other parameter, which (if sufficiently large) would be better tackled at the source. Phase error correction by magnetic shimming makes no distinction of the source of the error, however shimming might not always be an option (e.g., superconducting undulators), or (for example) the gap dependence may be different.

The three components of the phase error influence the radiation spectrum in different ways. In the cases examined phase oscillations due to trajectory deviations are relatively small and so have a relatively small effect on the spectral properties, thanks to the trajectory straightening that is usually part of the field optimization process. Whereas both the random and smooth components of the phase variation reduce the peak brightness, the smooth part also significantly broadens the brightness spectrum, which has the effect that the integrated flux (i.e., when including electron beam emittance and energy spread and finite collection angles) is not deteriorated to the same extent. This is the explanation for the observations made in [14], and earlier in [13], which were the initial inspiration for this work.

The reduction in peak brightness depends principally on the overall rms phase error, and follows Eq. (1) initially, independently of the number of magnet poles, but for larger errors and/or higher harmonics the effect of emittance and especially energy spread weaken the reduction. Despite this, the rms phase remains a good guide to performance, especially for reduction factors down to about 0.7 . The reduction in integrated flux on the other hand is not well correlated with the rms phase and instead the rms local phase variation (scaled in relation to the number of poles) is a better, or additional, figure of merit.

This analysis has also shown that the effect of phase errors will not be significantly different in lower emittance storage rings than current ones, the reason being that the emittance is already so low that the dominant factor is not actually the emittance but the energy spread.

\section{ACKNOWLEDGMENTS}

The author is greatly indebted to Sara Casalbuoni, for discussions about phase errors that led to the present work, Roger Dejus, for kindly providing phase error, spectral 
calculations, and other data for the APS and LCLS undulators that have been used in this report as well as responding to many questions, and Emily Longhi, for similarly providing information on the DLS insertion devices.

[1] B. M. Kincaid, J. Opt. Soc. Am. B 2, 1294 (1985).

[2] B. Diviacco and R. P. Walker, in Proceedings of the 1989 Particle Accelerator Conference, Chicago, IL (IEEE, New York, 1989), p. 1259.

[3] P. Kennedy et al., in Proceedings of FEL 1989 [Nucl. Instrum. Methods Phys. Res., Sect. A 296, 607 (1990)].

[4] B. L. Bobbs et al., in Proceedings of FEL 1989 [Nucl. Instrum. Methods Phys. Res., Sect. A 296, 574 (1990)].

[5] R. P. Walker, Nucl. Instrum. Methods Phys. Res., Sect. A 335, 328 (1993).

[6] G. Rakowsky, B. Bobbs, R. Burke, W. McMullin, and G. Swoyer, Nucl. Instrum. Methods Phys. Res., Sect. A 296, 597 (1990).

[7] B. Diviacco, in Proceedings of the Particle Accelerator Conference, Washington, DC, 1993 (IEEE, New York, 1993), p. 1590.

[8] J. Chavanne and P. Elleaume, in Proceedings of the 4th European Particle Accelerator Conference, London, UK, 1994 (EPS-AG, London, UK, 1994), p. 654.
[9] B. Diviacco and R. P. Walker, Nucl. Instrum. Methods Phys. Res., Sect. A 368, 522 (1996).

[10] S. C. Gottschalk et al., Rev. Sci. Instrum. 67, 3347 (1996) [http://dx.doi.org/10.1063/1.1147054].

[11] I. Vasserman, Report No. ANL/APS/LS-253, 1996.

[12] P. Illinski, R. J. Dejus, E. S. Gluskin, and T. I. Morrison, Proc. SPIE Int. Soc. Opt. Eng. 2856, 16 (1996).

[13] R. J. Dejus, Report No. ANL/APS/LS-304, 2003.

[14] S. Casalbuoni et al., in Proceedings of the International Particle Accelerator Conference, New Orleans, Louisiana, 2012, p. 711.

[15] R. J. Dejus, I. Vasserman, E. R. Moog, and E. Gluskin, Rev. Sci. Instrum. 66, 1875 (1995).

[16] I. Vasserman, R. J. Dejus, S. Sasaki, J.Z. Xu, E. M. Trakhtenberg, S. V. Milton, E. R. Moog, M. M. White, and N.A. Vinokurov, Nucl. Instrum. Methods Phys. Res., Sect. A 575, 22 (2007).

[17] J. Bahrdt and Y. Ivanyushkenov, in Proceedings of the International Particle Accelerator Conference, New Orleans, Louisiana, 2012, 708.

[18] R. J. Dejus and A. Luccio, Nucl. Instrum. Methods Phys. Res., Sect. A 347, 61 (1994).

[19] M. Eriksson et al., in Proceedings of the International Particle Accelerator Conference, San Sebastian, Spain, 2011, p. 3026.

[20] M. Borland, in Proceedings of the International Particle Accelerator Conference, New Orleans, Louisiana, 2012, p. 1035 . 